\title{
Aeroacoustic Coupling Effect during the Ascent of Space Transportation Systems
}

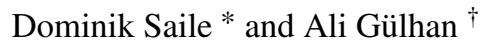 \\ Institute of Aerodynamics and Flow Technology, Supersonic and Hypersonic Technologies Department, Linder Hoehe, \\ 51147 Cologne, Germany.
}

After the failure of the maiden flight 157 of Ariane 5 ECA, the inquiry board responsible for the investigation reported as one of the probable causes the 'non-exhaustive definition of the loads to which the Vulcain 2 engine is subjected during flight'. As a result, many research activities were set up with the objective to isolate the driving mechanisms. Driving mechanisms such as pumping, flapping or swinging were found to excite oscillations in the base region. Despite this finding, the question why the pressure fluctuations and base flow excitations are especially pronounced in the subsonic to transonic flow regime remained unclear. This question is addressed in the study at hand. Results from literature are combined with recent wind tunnel measurements on the base model of a space transportation system. The measurement data were acquired by means of particle image velocimetry, pressure transducers and high-speed schlieren imaging. The results suggest that an aeroacoustic coupling takes place between a jet noise generation mechanism called screeching and the near-wake dynamics. Due to the nature of both effects, they are likely to reach a resonating frequency during the ascent. The resonant frequency appears to increase unfavorable fluctuations in the base region, which are held responsible for the amplified unsteady loads. A schematic model concept is proposed, which describes the underlying governing mechanism. It provides an explanation for question why buffeting effects are especially amplified in the high subsonic ambient flow regime.

\section{Nomenclature}

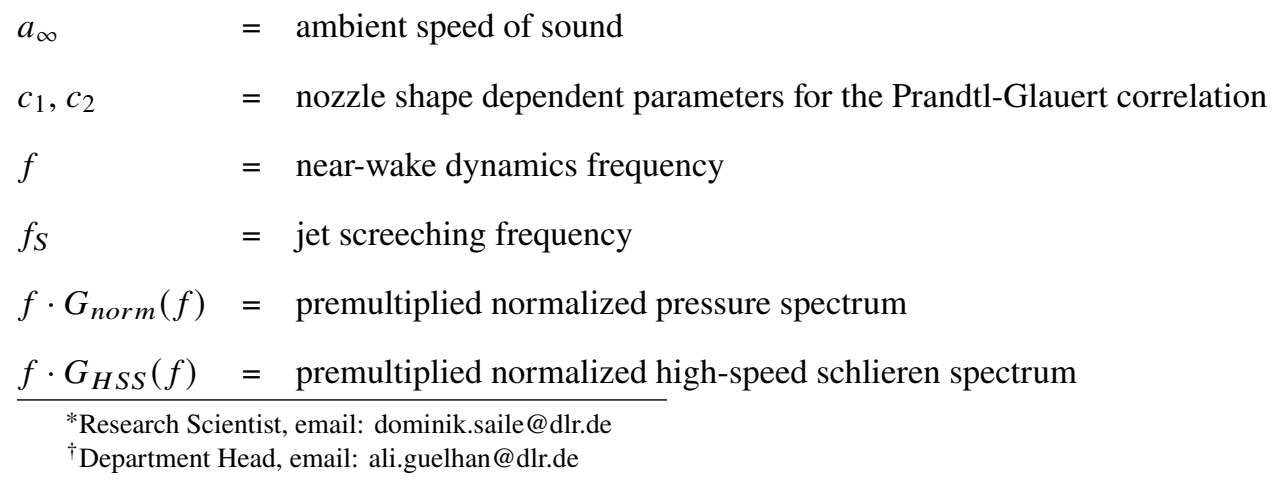




\begin{tabular}{|c|c|c|}
\hline$d_{\text {nozzle }}$ & $=$ & nozzle exit diameter \\
\hline$D$ & $=$ & base diameter \\
\hline$k s d$ & $=$ & kernel smoothing density \\
\hline$L_{c e l l}$ & $=$ & shock cell length of the jet \\
\hline$L_{R}$ & $=$ & shear layer reattachment lengt \\
\hline$M a$ & $=$ & Mach number of the ambient flow field \\
\hline$M a_{j}$ & $=$ & fully-expanded jet Mach number \\
\hline$p_{0}$ & $=$ & chamber reservoir pressure \\
\hline$p_{\infty}$ & $=$ & ambient pressure \\
\hline$p^{\prime}$ & $=$ & base pressure fluctuation \\
\hline$r$ & $=$ & coordinate in the radial direction \\
\hline$S r_{D}$ & $=$ & diameter D based Strouhal number \\
\hline$u, v$ & $=$ & streamwise, radial mean velocity component \\
\hline$u^{\prime}, v^{\prime}$ & $=$ & streamwise, radial velocity fluctuation component \\
\hline$<u^{\prime} u^{\prime}>$ & $=$ & average axial turbulence intensity \\
\hline$U_{\text {Conv }}$ & $=$ & convection velocity of the shear layer \\
\hline$U_{C}$ & $=$ & Wind tunnel nozzle exit velocity / reference velocity \\
\hline$U_{j}$ & $=$ & fully-expanded jet velocity \\
\hline$U_{\infty}$ & $=$ & ambient flow velocity \\
\hline$x$ & $=$ & coordinate in the axial direction \\
\hline$x_{v c}$ & $=$ & axial position of the vortex center location \\
\hline$\alpha$ & $=$ & phase angle for the description of a POD mode cycle \\
\hline$\zeta_{k, s t d}$ & $=$ & standard deviation of the time varying coefficient of the k-th mode \\
\hline$\kappa$ & $=$ & heat capacity ratio \\
\hline$\Psi_{k}$ & $=$ & POD basis function of the k-th mode \\
\hline
\end{tabular}

\section{Introduction}

FTER the failure of the maiden flight 157 of Ariane 5 ECA, the inquiry board responsible for the investigation is subjected during flight'. As a result, many research activities were set up with the objective to isolate the driving mechanisms.

However, before getting into the details of various base flow excitation mechanisms, the typical mean flow features of 


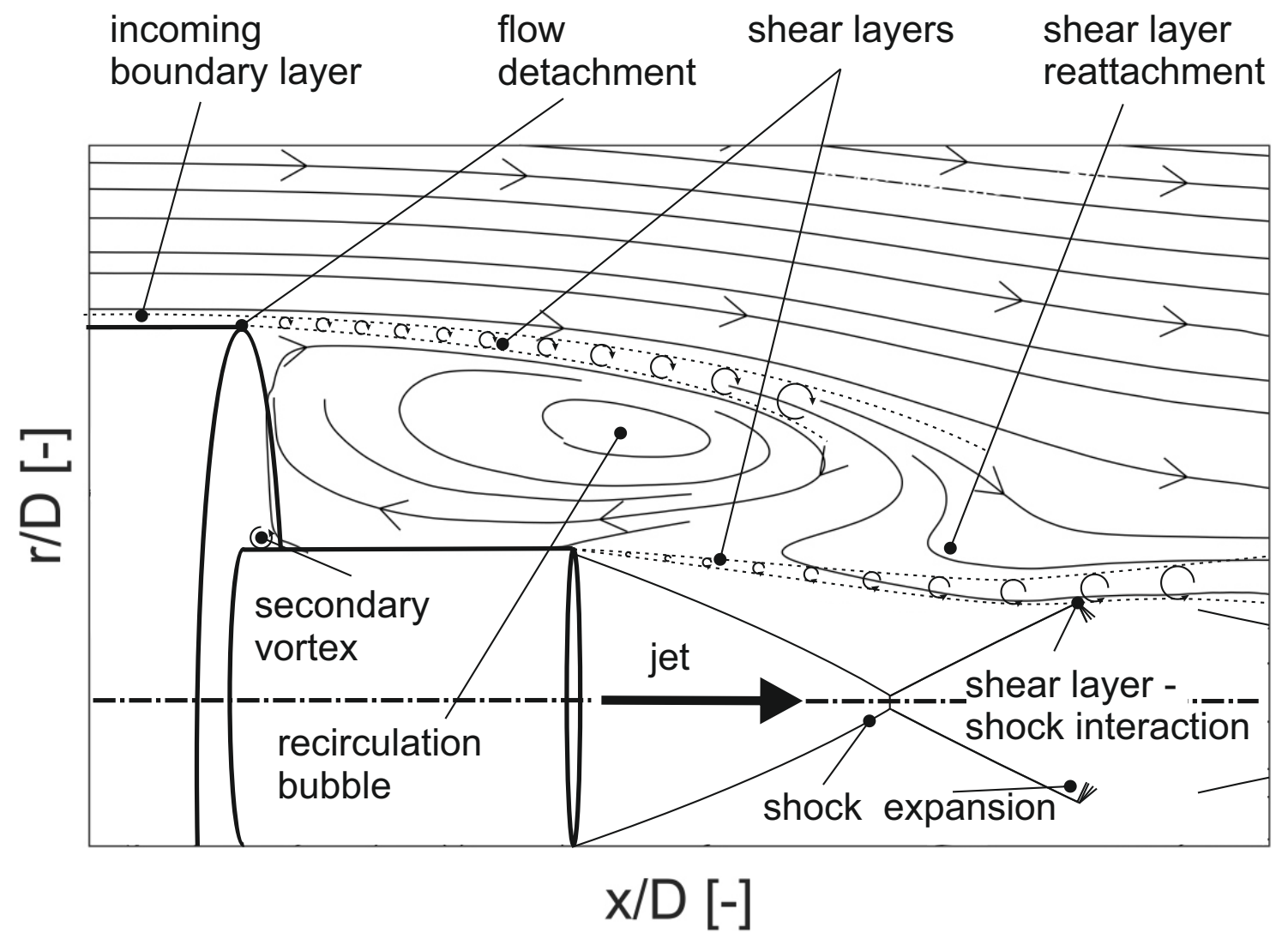

Fig. 1 Mean base flow topology and flow features in the subsonic wake of space launcher configuration with jet $[1]$.

a generic base configuration exposed to a high subsonic ambient flow are introduced in context of fig. 1. This schematic sketch [1] features an over-expanded, supersonic exhaust jet. It can be seen that the incoming flow separates at the shoulder of the wind tunnel model. From there, a free shear layer evolves, which impinges (for these conditions) farther downstream on the exhaust jet. As a result, the shear layer, the wall of the base, the wall of the nozzle surface and the jet enclose a recirculation region. On average, the recirculation region is dominated by a large vortex. Furthermore, a small corner/secondary vortex can usually be found between the base plate and the nozzle. Depending on configuration, the shear layer reattachment takes place exclusively on the jet (as shown in fig. 1), exclusively on the solid nozzle wall or is alternating between both options. In literature [2], the latter is referred to as a hybrid reattachment.

Turning now to the nature of the dominant motions, which is important regarding the induction of loads. The relevance of the buffeting issue for Ariane 5 is shown by in-flight measurements in the study by David and Radulovic [3]. A strong amplification of the pressure oscillations and interrelated loads on the actuator acting on the nozzle gimbal occur for an ambient flow at Mach 0.8. These oscillations act with a diameter-based reduced frequency of $S r_{D} \approx 0.2$. Schwane [4] reports of pressure fluctuations with increasing amplitude in the first $37 \mathrm{~s}$ of the Ariane 5 Flight A501 ascent, meaning these fluctuations occur in the transonic flow regime of Ariane 5. Note that the definition of the reduced 
frequency, or Strouhal number $S r$, is based on the frequency $f$ of a near-wake excitation, the base diameter $D$, and the ambient flow velocity $U_{\infty}$. The equation is provided in eq. (1).

$$
\operatorname{Sr}_{D} \equiv \frac{f \cdot D}{U_{\infty}}
$$

On the search for the origin of the excitations, Statnikov et al. [5] focused on the identification of modes by means of a dynamic mode decomposition (DMD) for a space launcher configuration with ambient flow and jet. The study reveals mainly four different interacting phenomena: Cross-pumping, vortex shedding, cross-flapping and shear layer swinging. The term cross refers to the three-dimensional distribution of the corresponding mode, e.g. a wavy pattern in the cross direction as the azimuthal or spanwise direction [6]. A schematic visualization of the motions is provided in fig. 22 This is the schematic sketch synthesized from the results in Statnikov et al. [6] and the POD analysis of the comparable data set presented in Saile [1]. It shows that cross-pumping captures an overall growth and decay process of the recirculation bubble, cross-flapping the overall flapping motion of the shear layer, while swinging reflects the intrinsic twist of the shear layer and vortex shedding the shedding of a vortex from the rear end of the nozzle.

In the work by Statnikov et al. [5], more details are attributed to the nature of the modes. There, cross-pumping describes a harmonic antisymmetric oscillation of the main recirculation vortex taking place with a reduced frequency of $S r_{D} \approx 0.1$. Vortex shedding is interrelated with the former cross-pumping motion. The antisymmetric growth and decay process of the recirculation bubble triggers in-phase and twice per cross-pumping cycle the shedding of a vortical structure from the nozzle end, meaning it happens twice as often with a reduced frequency of $S r_{D} \approx 0.2$. The vortex shedding process in turn causes or is connected with the in-phase cross-flapping motion of the shear layer, which equally features a periodicity of $S r_{D} \approx 0.2$. Last, shear layer swinging is the result of a higher harmonic of the vortex shedding mode acting with $S r_{D} \approx 0.35$.

Note that the work by Statnikov et al. [5] represents one of the latest publications. Before, the studies by Deprés et al. [7], Nguyen et al. [8], Wolf [9], or Schrijer et al. [10] contributed significantly to the issue of modal decompositions of base flows. Meanwhile, due its generic similarity to the European Ariane 5 launcher, the investigated configuration can be considered as standardized test case. This or similar test cases were used in a couple of programs such as in the frame of the FESTIP: Future European Space Transportation Investigation Programme of the ESA-TRP Unsteady Subscale Force Measurements within a Launch Vehicle Base Buffeting Environment [11] and of the DFG-TRR40 Fundamental Technologies for the Development of Future Space-Transport-System Components under High Thermal and Mechanical Loads [12] project resulting in a solid number of publications. The publications listed next are limited to the ones focusing on subsonic ambient flows investigating (pseudo-)axisymmetric base configurations. Among others, corresponding experimental work was executed by Saile [1], van Gent et al. [2], Deprés et al. [7], Wolf [9], Schrijer et al. [10], Lê [13], Schrijer et al. [14], Gentile et al. [15, 16], van Gent et al. [17], Saile et al. [18, 19, 20] without or with 


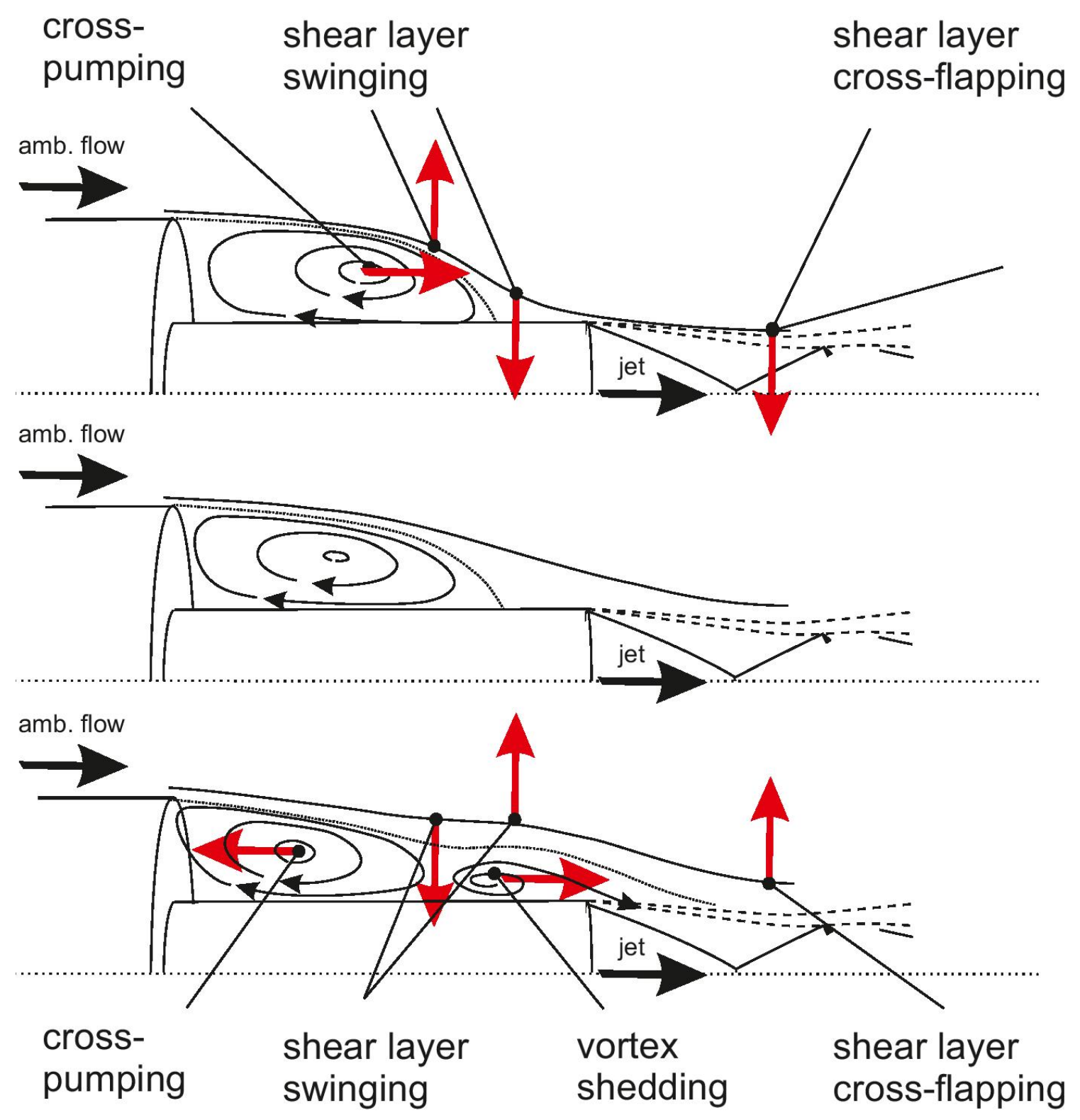

Fig. 2 Schematic of the dominant base flow modes based on a reconstruction of the first POD mode (synthesis of the experimental results by Saile [1] with numerical results by Statnikov et al. [5]). Depicted are the mean flow topology (mid) and the two reversal points of a hypothetical POD cycle (top/bottom). 
cold exhaust jet. The impact of a hot jet has lately been addressed by Kirchheck et al. [21]. Insights from the numerical point of view is provided Deck and Thorigny [22], Weiss et al. [23], Weiss and Deck [24], Pain et al. [25, 26].

The activity here obviously targets the transfer of the findings to a large scale application, thus similarity aspects must be considered. For the configuration at hand, this was already addressed in previous publications of that series [1, 18-20]. In short, it was found that the effect of the wind tunnel environment appears to be negligible with respect to the mean and turbulent velocity flow field [1, 18]. CFD simulation on that issue under comparable conditions come to the same conclusion Banuti [27]. Reynolds number effects appear to be small for the investigated (diameter-based, free-stream) Reynolds number range between $0.8 \times 10^{6}$ and $1.6 \times 10^{6}$. This seems reasonable, since, on the one hand, this regime falls into the category of trans-/postcritical near-wake flows with similar effects according to the categorization of Morkovin [28]. On the other hand, for flows over a backward-facing step, similar flow fields are expected [29-33] as long as the boundary layer can be considered as 'thin', meaning that the ratio between the boundary layer thickness and the step is smaller than 0.4. This is the case for the experiments at hand $(\approx 0.07)$ as well as for Ariane $5(\approx 0.09)[19]$. Other similarity parameters concern the exhaust jet. Here, similarity with respect to the displacement effects controlled by the plume topology can be approximated. Ariane 5 as well as the current test case feature an overexpanded nozzle flow due to a reasonably comparable pressure ratio between the nozzle exit and the ambient flow of 0.25 and 0.36 (e.g. for the Mach 0.8-case), respectively. Nevertheless, the jet poses the largest deviation to a real-flight configuration mostly due to the temperature difference and the usage of air, which is interrelated with many other properties such as the exit velocity, density, viscosity, ratio of specific heats. To compensate for the latter, Goethert and Barnes [34] suggested to keep $\kappa M a^{2} / \sqrt{M a^{2}-1}$ similar between the test configuration and the full-scale application for the nozzle flow. Here, this corresponds to 5.2 vs. 5.6, respectively. More difficulties arise with the other properties since they significantly influence the evolution of the shear layer, and in that sense, the cold and hot cases differ. As impact, one would expect a smaller recirculation region due to a pressure drop induced by an increased entrainment of mass from the ambient flow field into the shear layer. However, this was not necessarily observed in Kirchheck et al. [21], and thus, it is still under investigation.

Next, the counterpart for the hypothesized aeroacoustic feed-back mechanism is introduced, namely the jet generation mechanism called screeching [35-37]. A schematic sketch is depicted in fig. 3. It shows the essential four screeching elements as found by Powell [38, 39]: (1) The growth of instability wave in the supersonic shear layer of the jet, (2) the interaction of the turbulent coherent structures with the shock-cells, (3) the upstream propagation of acoustic waves and (4) the closure of the feedback loop by converting acoustic waves to instability waves at the nozzle tip region. Tam et al. [40] suggested the nozzle tip as especially receptive for excitation and it is therefore considered as weakest link for the feedback of information in the screech loop. Obviously, the receptivity of the acoustic waves is essential for the aeroacoustic coupling.

In a screech cycle, the interaction between turbulent coherent structures and the shock-cell (2) is responsible for 


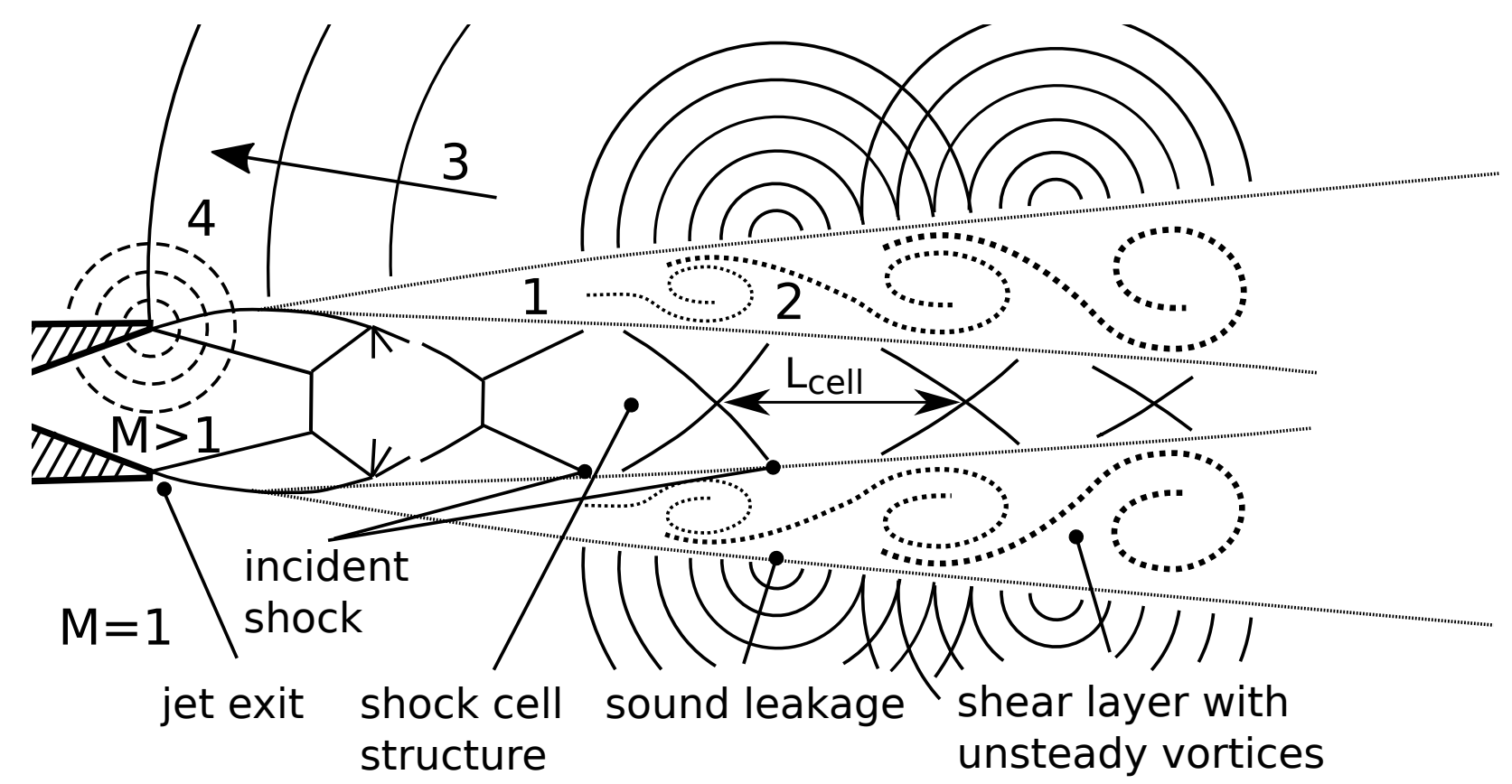

Fig. 3 Schematic of the screeching jet noise generation mechanism (extracted from reference [1] and based on the visualization of Suzuki and Lele [42])

noise generation process. In numerical simulations, Manning and Lele [41] found an unsteady refraction of the incident shock of the shock-cells in the shear layer in between the passage of vortices, which results shock leakage (from the jet). Thus, Manning and Lele [41] suggest shock leakage as source for the release of high amplitude waves.

The frequency $f_{S}$ of the high amplitude waves resulting from the passage of vortices in the shear layer through quasi-periodic shock-cells can be determined by means of eq. (2) as extracted from Wolf [9]. It can be seen that the shock cell length $L_{c e l l}$, as visualized in fig. 3 is one of the defining parameters. Furthermore, the screeching frequency must be compensated for the current in-flight conditions. On the one hand, this is done by reducing the speed of sound $a_{\infty}$ (responsible for upstream propagation of the acoustic disturbance) by the ambient flow velocity $U_{\infty}$. On the other hand, it must also be incorporated for the downstream convection velocity of the instability in the shear layer $U_{C o n v}$ as done in eq. (3) [9, 43]. The quantity $U_{j}$ reflects the fully-expanded jet velocity.

$$
\begin{gathered}
f_{S}=\frac{U_{\text {Conv }}}{L_{\text {cell }}\left(1+\frac{U_{\text {Conv }}}{a_{\infty}-U_{\infty}}\right)} \\
U_{\text {Conv }}=0.7 \cdot\left(U_{j}-U_{\infty}\right)+U_{\infty}
\end{gathered}
$$

Another fully-expanded quantity, the Mach number $M_{j}$, is used to determine the shock-cell length $L_{c e l l}$ as ratio to the nozzle exit diameter $d_{\text {nozzle }}$. According to Seiner and Norum [44], the shock cell length can be empirically determined by means of the Prandtl-Glauert correlation as given in eq. (4). The empirical parameters $c_{1}$ and $c_{2}$ are 
dependent on the individual nozzle shape. The fully-expanded Mach number $M_{j}$ in eq. (5) is computed by means of the reservoir pressure of the chamber $p_{0}$, the ambient pressure $p_{\infty}$, and the heat capacity ratio $\kappa$. Note that these equations are used later for comparisons with the measured frequencies (fig. 11) and for the prediction in context of Ariane 5 (fig. 16.

$$
\begin{gathered}
\frac{L_{\text {cell }}}{d_{\text {nozzle }}}=c 2 \cdot\left(\sqrt{M a_{j}^{2}-1}\right)^{0.5 \cdot c 1} \\
M a_{j}=\sqrt{\frac{2}{\kappa-1}\left(\left(\frac{p_{\infty}}{p_{0}}\right)^{\frac{1-\kappa}{\kappa}}-1\right)}
\end{gathered}
$$

In flight-screeching is a sparsely treated topic. More details can be found in Wolf [9], Hay and Rose [45], Bryce and Pinker [46], Adrian [47], which is from where the current information was extracted. More details to screeching or jet noise and the progress made over the years are documented in the review papers of Raman [37] and Jordan and Colonius [48]. Further, remarkable videos on the screeching issue can be found in the scientific films shot by Poldervaart et al. [49] and Poldervaart et al. [50].

\section{Methods}

The current study is treated as summarizing review on the hypothesis of an aeroacoustic coupling effect reported in the doctoral thesis in Saile [1]. This hypothesis was developed in the frame of wind tunnel experiments in the Vertical Test Section Cologne (VMK) [51, 52] by means of particle image velocimetry, base pressure transducers and high-speed schlieren imaging. Parts of the doctoral thesis have been extracted and published separately in Saile [1], Saile et al. [18, 19, 20]. Thus, it is recommended to take further technical details either from the corresponding publication or the doctoral thesis.

Nevertheless, the key parameters are provided in the following. For particle image velocimetry, a classical 2D-2C setup was applied, while the flow was seeded with titan dioxide particles. Particle images of an overall size of $134 \times 100 \mathrm{~mm}^{2}$ were captured with a recording frequency of $15 \mathrm{~Hz}$. The evaluation was executed with an interrogation window of the size $32 \times 16 \mathrm{px}\left(2.68 \times 1.34 \mathrm{~mm}^{2}\right)$ with an overlap of $4 \times 4 \mathrm{px}$. On average, the $95 \%$ confidence level was in the range of $\pm 2 \%$ including the uncertainties related to the equipment, to the particle dynamics and to sampling. The base pressure was acquired with a recording frequency of $17.0 \mathrm{kHz}$ by means of flush-mounted XCQ-080 Kulite transducers. This type of sensor exhibits an error of $\pm 0.1 \%$ for a best fit straight line. The density gradients were captured with the same recording frequency by means of a Photron FASTCAM-APX RS camera. For that purpose, a Z-type schlieren path setup [53] is available in VMK.

The temporally highly-resolved schlieren recordings are used to assess the mean and unsteady properties in the 
density gradient field. To deduce information about the spectral content, each pixel is treated as individual sensor on which a spectral analysis according to Welch [54] is applied. Afterwards, the spectrum is averaged over the complete field of view. The spectral analysis for the pressure signal is also based on the approach by Welch [54].

The subsonic tests ranging from Mach 0.5 to 0.9 were executed in VMK, which features an open test section. Correspondingly, the free-stream pressure is equal to the ambient pressure and the reservoir pressure must be set accordingly to reach the desired Mach number. Further, the tests were run unheated, meaning the reservoir temperature corresponds to the ambient temperature. The wind tunnel model is a generic representation of the base geometry of the main core of Ariane 5 (e.g. fig. 11). In other words, the outer geometry resembles two cylinders stacked on top of each other while the cylinder with the larger diameter $(D=66.7 \mathrm{~mm})$ mimics the main core and the attached smaller cylinder $(d=26.8 \mathrm{~mm})$ contains a conical nozzle contour on the inside with an expansion ratio of $\epsilon=7.37$. The length of the nozzle (outer geometry) was varied for some tests between $L / D=0.45$ to $L / D=1.2$ [20]. However, typically it is set to $L / D=1.2$ to resemble the main outer features of Ariane 5. For the jet:off-case, the nozzle is plugged, while for the jet:on-case, it is fed with a reservoir pressure of 3.3 MPa from a reservoir staying at ambient temperature.

The publications mentioned above have base flow issues of a blunt rocket base in common. However, the various publications focus on different aspects. In detail, the series can be differentiated as following: Reference [18] considers base flows without an exhaust jet for a subsonic ambient flow; then, reference [19] introduces a cold, overexpanded, supersonic exhaust jet for subsonic ambient flows; reference [20] investigates the impact of a nozzle length reduction for Mach 0.8; reference [1] contains a POD decomposition (as shown in the references [8--10, 55]) on the previously acquired velocity fields and the fluctuations of the pressure and high-speed schlieren recordings with focus on their spectral content is also evaluated there.

\section{Results}

This section first reviews elements of previously published findings (section III.A] of this series [1, 18, 20] for completeness and as background information before moving on to the new contributions (section [II.B.

\section{A. Review}

In the current series, the base flows without an exhaust jet for a subsonic ambient flow was investigated in Saile et al. [18]. There, self-similar features were found in the velocity and velocity fluctuations distribution for the current configuration if scaled with the reattachment length of the shear layer. For a backward-facing step and for blunt base configurations, self-similar features have already been observed by Roshko and Lau [29], Westphal et al. [30], Merz et al. [31], Adams and Johnston [32], Nadge and Govardhan [33], Uberoi and Freymuth [56].

In Saile et al. [19], the same configuration was addressed with a supersonic exhaust jet. It was found that the mean velocity distribution scales reasonably well with the reattachment length for all Mach numbers. No peculiarities were 


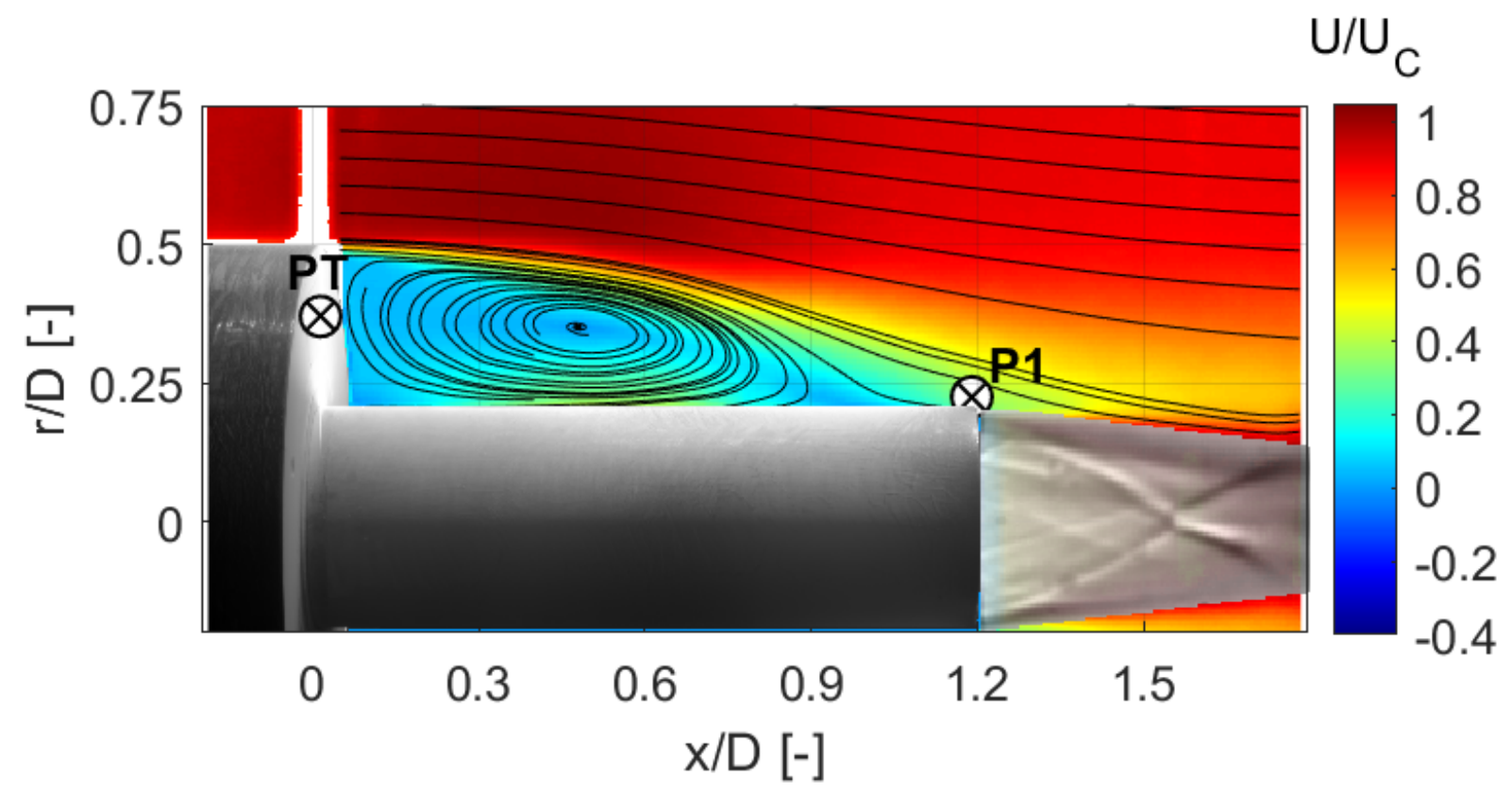

Fig. 4 Contour plot with streamlines of the mean velocity field for $M a \sim 0.8$ [19]. The point 'P1' marks a velocity probe location used in context of the reattachment location (see fig. 7); 'PT' denotes the location of the pressure transducer used for the spectra in fig. 11 .

observed in that quantity as it can be seen in the mean velocity field in fig. 4 acquired by means of PIV for the Mach 0.8-case. The mean flow field exhibits the typical features of a base flow of a space transportation system. The incoming flow separates from the main body at the shoulder from where a free shear layer evolves. Here, the free shear layer reattaches on average on the nozzle and encloses a recirculation bubble. Farther downstream, the overexpanded jet entrains air from the ambient flow. Regarding the mean flow field, there appears to be no significant difference to the base flows for higher or lower subsonic Mach numbers [19].

In contrast to the mean flow field, an outstanding deviation to the other Mach numbers was found for the turbulence intensity distribution at Mach 0.8 , which is visualized in fig. 5 As expected, the distribution shows areas of increased turbulence intensity in the shear layer, but also another strong area inside the recirculation bubble in the vicinity of the vortex center. The origin of the latter was attributed to the unsteady 'dancing' motion of the vortex center [19].

The radial profile slices in fig. 6 through that high turbulence intensity region at $x / L_{r}=0.61$ show that this Mach 0.8-case appears to be special indeed. While the turbulence intensity profiles (scaled with the reattachment length) agree for all Mach numbers with a reasonable degree, a totally different behavior manifests for Mach 0.8: For this case, exceptionally strong fluctuations can be observed inside the recirculation bubble (denoted here as anomaly) leading to the question why this condition is special.

The probability density distribution of the axial velocity component just at the tip of the nozzle (fig. 7) sampling location marked as 'P1' in fig. 4) evidences first clues on why the Mach 0.8-case is special and behaves differently. 


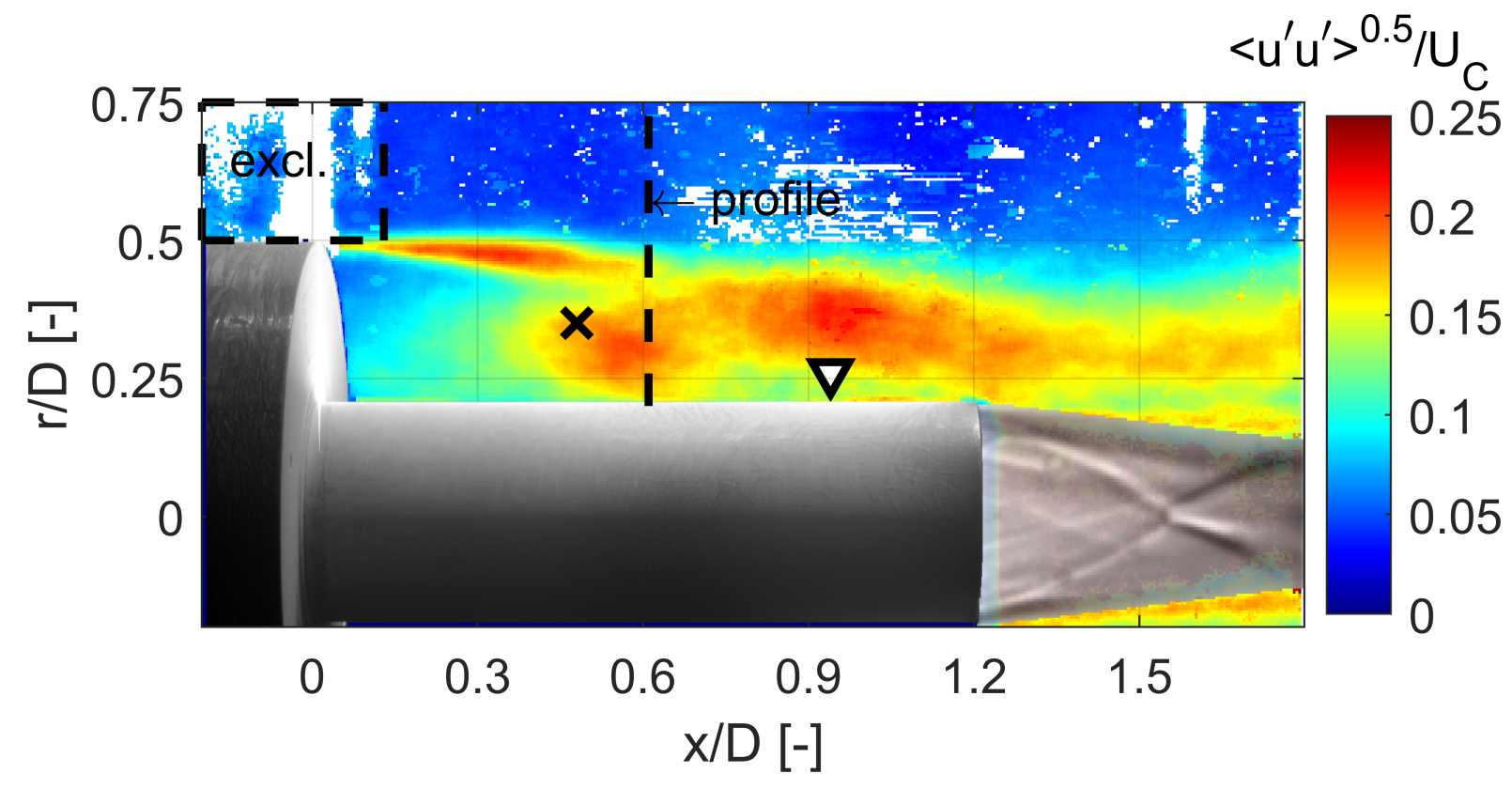

Fig. 5 Contour plot of the normalized axial turbulence intensity field for $M a \sim 0.8$ [19]. The center of the main vortex and the reattachment location are plotted as cross $\times$ and as triangle $\nabla$, respectively. The dotted line labeled as 'profile' marks the profile location used in fig. 6. The box labeled with excl. referring to 'excluded' is to be ignored due to errors induced by laser light reflections.

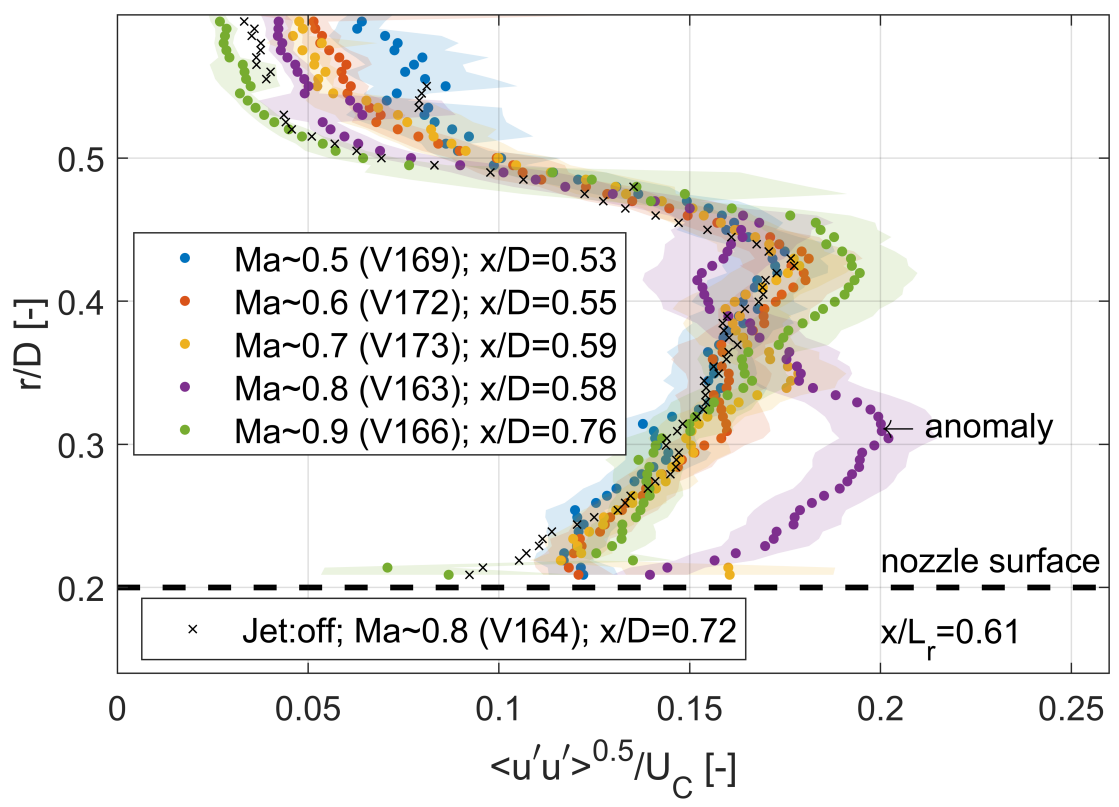

Fig. 6 Normalized axial turbulence intensity profiles for the downstream location $x / L_{r}=0.61$ (see fig. 5). The shading reflects the uncertainty [19]. 


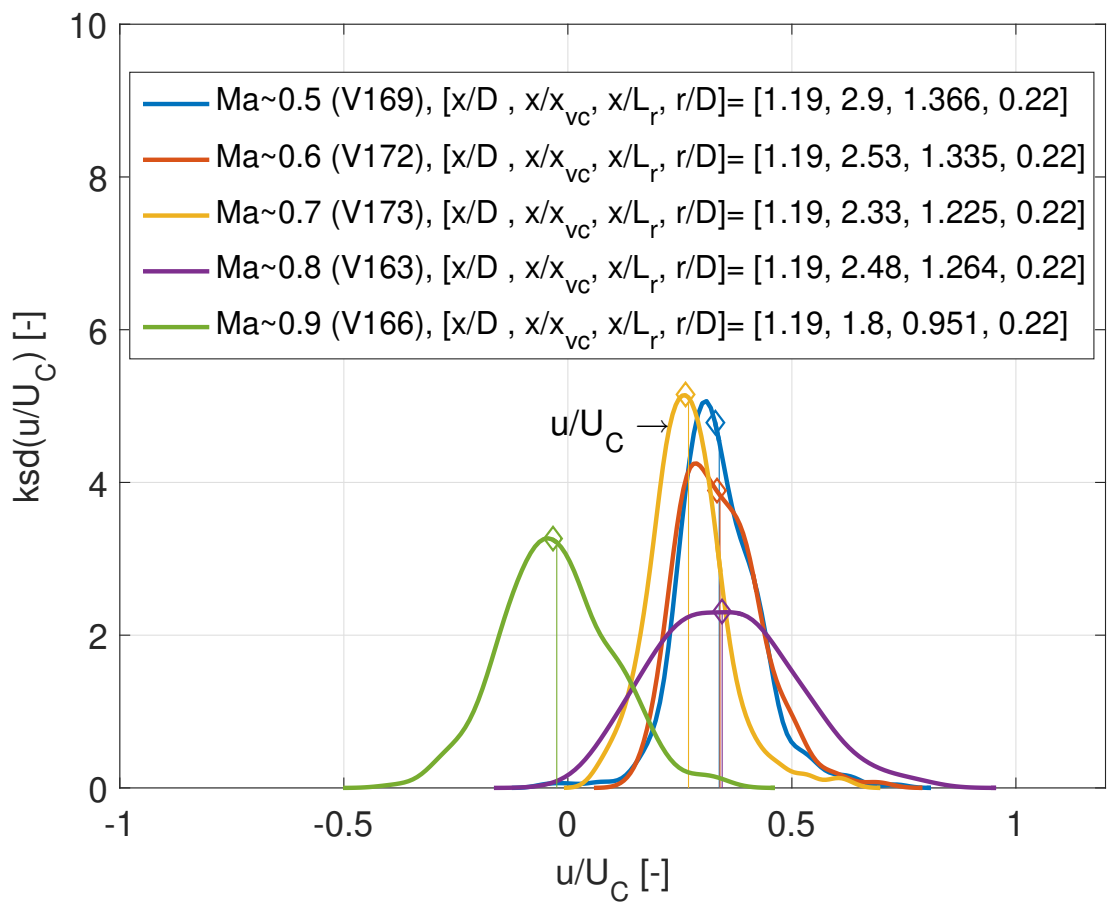

Fig. 7 Probability density estimate (as kernel smoothing density ksd) of the axial normalized velocity sampled upstream from the nozzle exit at point P1 at $[x, r] / D=[1.19,0.22]$ (see fig. 4) [19]. Diamond $\diamond$ markers denote the normalized mean axial velocity; the thin continuous line the median velocity.

For Mach numbers $\leq 0.7$, the axial velocity component only takes positive values, meaning that the reattachment predominantly takes place on the nozzle wall. For Mach 0.8 , a broadening of the distribution can be detected. On the one hand, this indicates fluctuations over a wider velocity range, and on the other hand the values close to zero suggest that the recirculation bubble is temporally connected with the jet. The recirculation bubble and the jet can now directly interact, meaning the reattachment process is of hybrid nature. A hybrid reattachment becomes certain for Mach 0.9: Up- and downstream pointing axial flow velocity can both be found in the distribution.

NOte that for the proposed and discussed resonant case between near-wake dynamics and screeching, the effect of the reattachment process on the turbulence intensity was investigated systematically in reference [19] by means of shortening the nozzle length for the Mach 0.8-case. Under these circumstances, it was found the turbulence along the shear layer is the strongest when the mean reattachment length is just at tip of the nozzle.

\section{B. Current Contributions}

The unusually high turbulence intensity already presented above is now scrutinized in context of the pressure-time series taken with transducers on the base plate of the model, which is shown in fig. 8. A stark contrast of the pressure series characteristics for the various configurations can be observed. For the near-wake flow case without jet [18], periodic pressure oscillations can be found to a small degree. However, these oscillations seem to be negligible and 


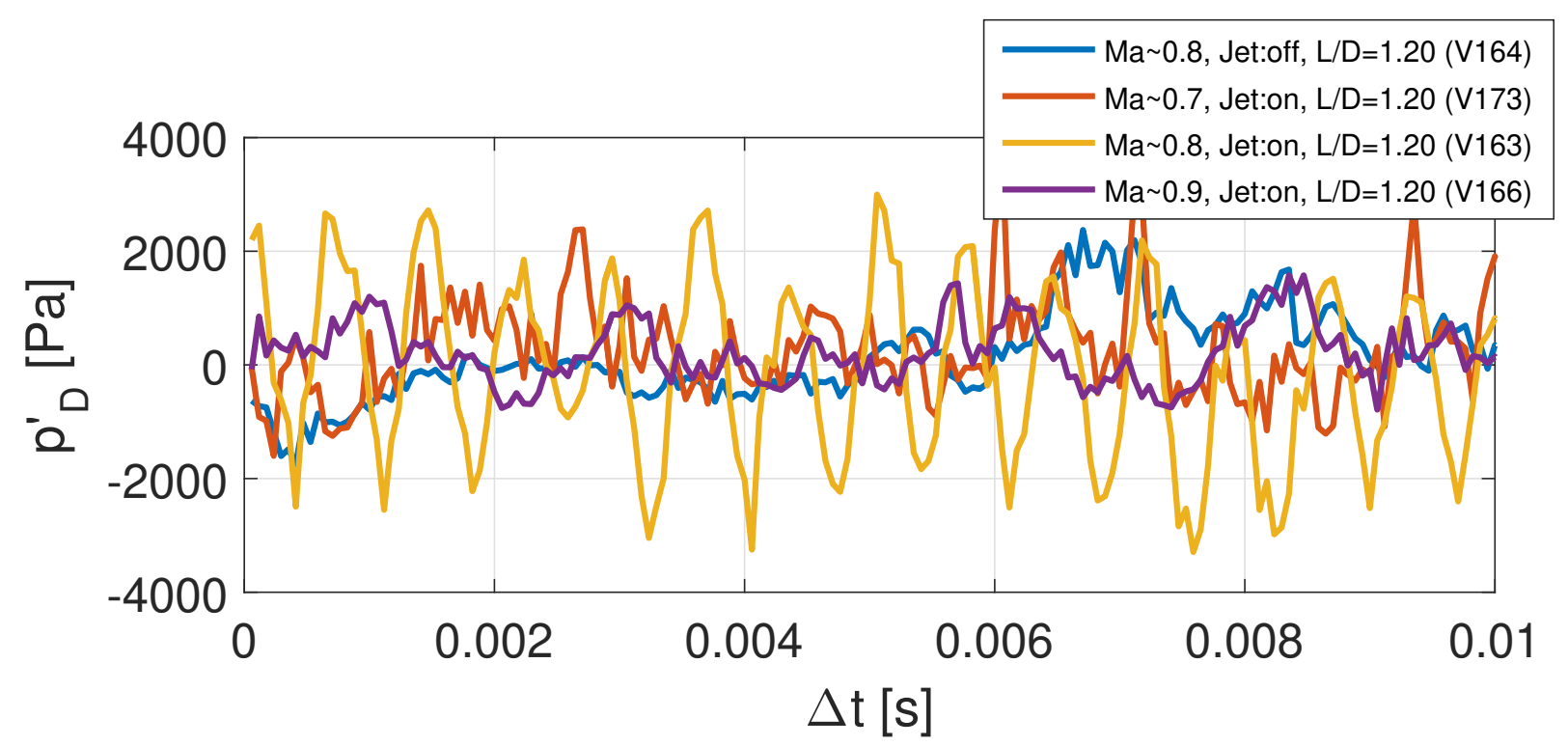

Fig. 8 Pressure time series of a selected pressure transducer on the base (data extracted from reference [1]). Data is provided for Mach $0.7,0.8$ and 0.8 with jet (jet:on), and additionally for Mach 0.8 without jet (jet:off). The location of the pressure transducer is marked as 'PT' in fig. 4 .

the pressure-time series appears to be dominated by stochastic fluctuations. When the jet is turned on, this changes drastically for an ambient flow at Mach 0.8: The results show highly coherent periodic oscillations fluctuating with seemingly one dominant frequency. For incrementally decreased and increased Mach numbers, this periodicity is not as prevalent anymore.

As last measurement method apart from PIV and pressure measurements, high-speed schlieren measurements were conducted focusing on the exhaust of the cold, overexpanded, supersonic jet. Figure 9 shows such a schlieren image at one instance in time. It shows the typical features of an overexpanded jet such as shock structures or the turbulent shear layer at the boundary of the jet. However, what is more interesting in the context of this study is the shock-like structure/acoustic upstream-traveling wave (UW) marked as yellow dotted line. This wave is observable on both sides of the nozzle, meaning in that case, this effect is axially symmetric. Further, when a sequence of images is considered, one can see that this shock-like structure travels periodically upstream towards the base. Such upstream traveling waves evidence a possible jet noise screeching mechanism. Keep in mind that screeching can also exhibit other than symmetric modes [38, 57].

The instantaneous high-speed schlieren sequence shown in fig. 10 reveals further remarkable aspects. First, the previously described upstream traveling wave can be detected for that Mach 0.8-case with exhaust jet. Next, this feature seems to be related to a downstream-transported feature denoted as VS1 and VS2. Taking into account that vortex shedding is a commonly described effect in turbulent near-wake flows [28], that the observed patches appear periodically just as observed for shear layer flapping and vortex shedding [5], these density gradient patches presumably reflect a 


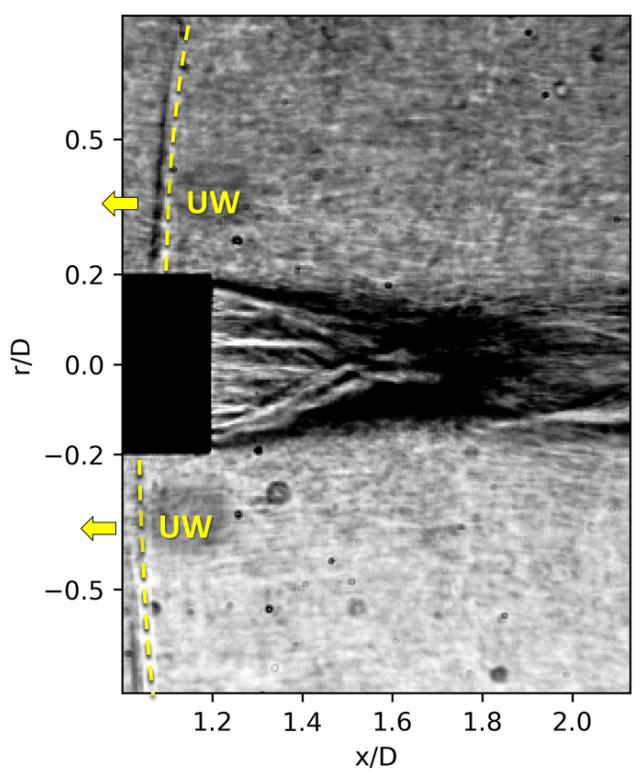

Fig. 9 Instantaneous high-speed schlieren image with focus on the visualization of the occurring upstream traveling wave (UW), which is highlighted as yellow, dotted line.

vortical structure (VS) resulting from the vortex shedding process of the shear layer. In other words, this sequence suggests a coupling between the upstream-traveling wave induced by screeching and downstream-traveling vortex. The latter appears to be shed from the near-wake, or more specific, from the shear layer.

Watching the high-speed schlieren video, this process appears to be periodic. The pressure time-series above in fig. 8 also evidence periodicity. Thus, both measurement results are analysed with respect to their spectral content to extract the dominant frequencies. The result of which is shown in fig. 11 for the transducer on the base plate and the high-speed schlieren signal (HSS). Note that the focus of this graph lies on the frequency and not on the amplitude which is the reason why the amplitude of the peak at $S r_{D}=0.18$ is not fully resolved.

Oscillations are notable at a non-dimensional frequency of $\operatorname{Sr}_{D}=0.11,0.18$ and 0.35 , which are typically associated with the base flow modes of pumping, flapping and swinging [5]. When the jet is turned on, one can detect a large peak at the frequency attributed to the latter mode. This peak is notable in both signals, the pressure and the high-speed schlieren signal. Further, the investigations on the impact of various relative nozzle lengths $L / D$ [1, 20] showed that this peak appears to stay at about the same frequency as long as $L / D$ is larger than 0.45 . For $L / D=0.45$, the separated shear layer overshoots the nozzle completely, meaning it exclusively reattaches on the jet. In other words, an oscillation at this frequency is only present for the investigated configurations with $L / D \geq 0.6$, which are the configurations for which a hybrid reattachment can be found.

Additionally, the spectra appear to exhibit peaks at higher harmonics as it often can be found for screeching. For this reason, the calculated in-flight screeching frequency is plotted for three different shock cell lengths. Unfortunately, 

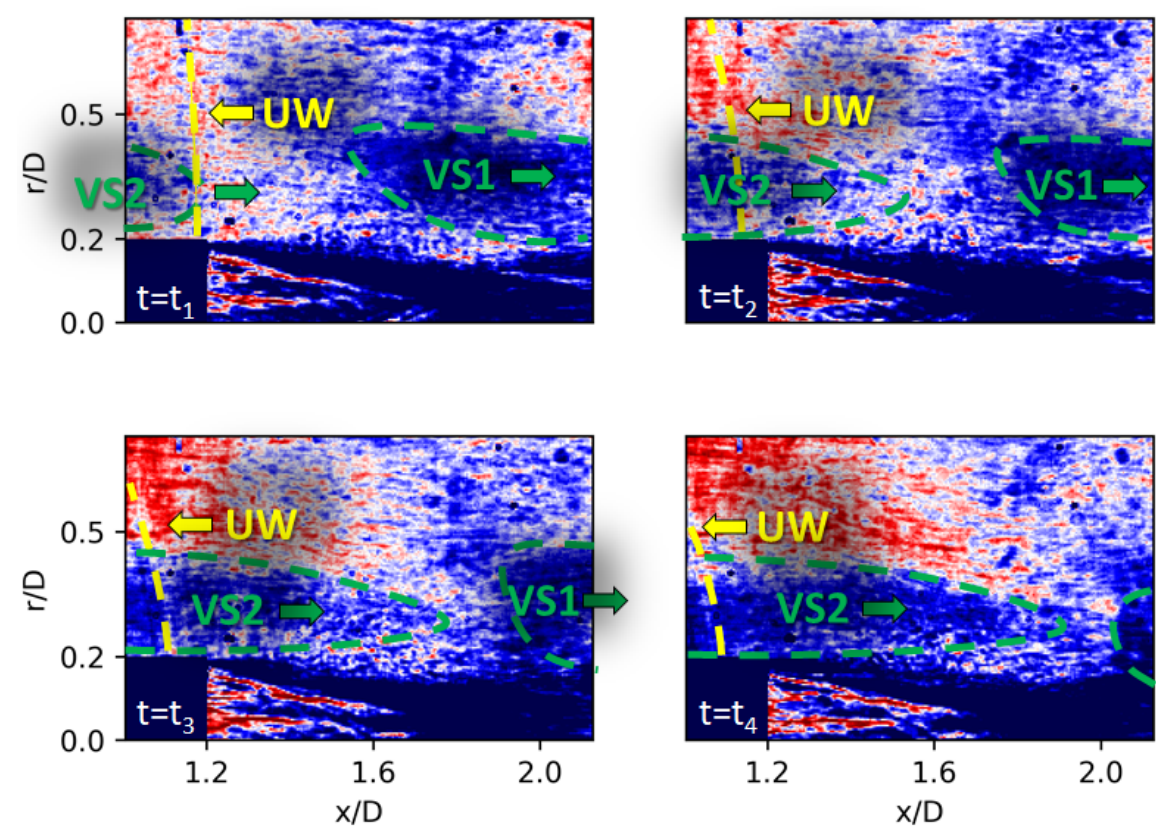

Fig. 10 Sequence of false-colored, instantaneous high-speed schlieren images highlighting the downstream transportation and shedding two vortical structures (VS1 and VS2, respectively) in combination with an upstream traveling wave $(\mathrm{UW})$. The time interval between the images is equivalent to the recording frequency of $17 \mathrm{kHz}$.

due to the restricted field of view, only the first shock cell of the jet could actually be determined from the high-speed schlieren recordings [1]. This shock cell exhibited a length of $52 \mathrm{~mm}$. Nevertheless, it is assumed that the successive shock cells have a shorter length since the incident shock of the jet shock train (see fig. 8) is reflected as expansion wave at the shear layer at a smaller radial distance due the growth of the jet shear layer in the downstream direction. Additional measurements with Mach numbers in the range between 0.5 and 0.9 in reference [1] showed the same tendency, meaning a good prediction for in-flight screeching frequency on the basis of the measured shock-cell length. Consequently, the graph suggests that the swinging mode as part of the near-wake dynamics oscillates with the same frequency as predicted for screeching. Thus, this appears to be a resonating case, and is consequently seen as one of the possible reasons for the previously found anomalies.

One interesting question now is how the dynamics of the near-wake flow changes with the presence of a jet. This question is assessed in reference [1] with a proper orthogonal decomposition (POD) [8, 10, 55]. An excerpt of the most dominant (first) POD mode is shown for the case without and with jet in fig. 12 and fig. 13 , respectively, for an ambient flow at Mach 0.8 .

For the case without jet (fig. 12), the first POD mode is dominated by a large coherent structure that causes an acceleration of the mean flow 'downstream-towards the axis' or in the reverse direction, which is 'upstream-in the radial direction'. This POD mode is responsible for about $13 \%$ of the near-wake energy; for the second POD mode, it is about 


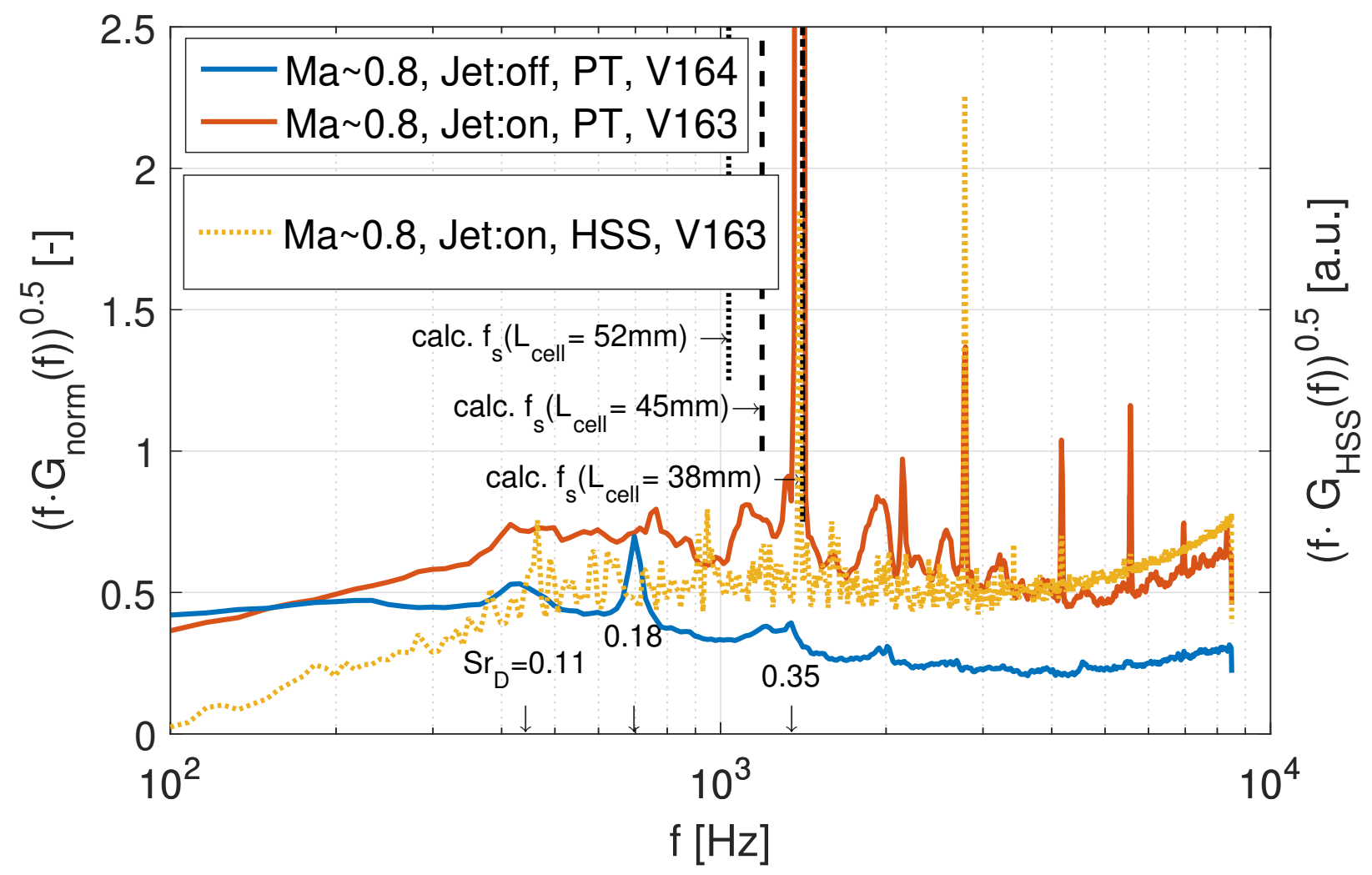

Fig. 11 Premultiplied normalized base pressure (PT) and high-speed schlieren (HSS) spectra for an ambient flow at Mach 0.8 (extracted from reference [1]). The pressure spectra are shown for the jet:off and jet:on-case. Further, the calculated screech frequency is incorporated for a shock cell length of $L_{c e l l}=52,45$ and $38 \mathrm{~mm}$. 


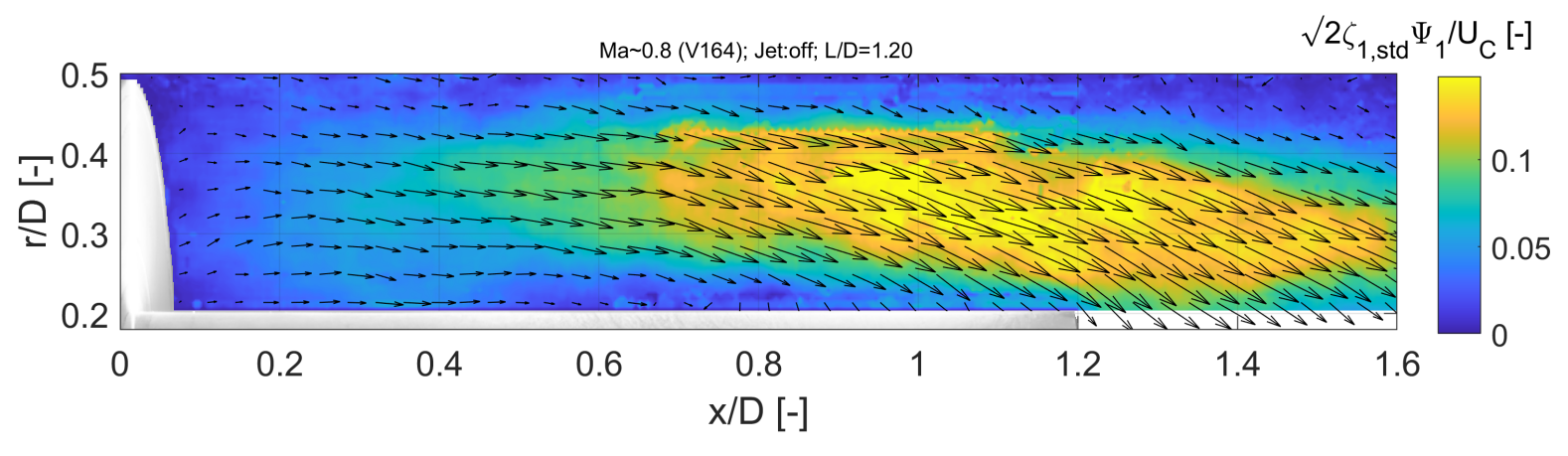

Fig. 12 First POD-decomposed velocity mode (basis function $\Psi_{1}$ scaled with the standard deviation of the time varying coefficient $\left.\zeta_{1, s t d}\right)$ for the configuration without jet $(L / D=1.2)$ for an ambient flow at Mach 0.8 [1]. The vector field and the scalar field show the components of the POD-decomposed velocity fluctuation and their absolute value, respectively.

$8 \%$ [1]. Further, it is shown in reference [1] that this coherent structure persists as first POD mode over the Mach number range from 0.5 to 0.9 . The most prevalent influence of increasing Mach numbers is a general downstream shift of that coherent structure, meaning a downstream shift of the region where the most dominant velocity fluctuations take place.

When the jet is turned on, the dynamics in the near-wake does not significantly change for most Mach number cases [1]. In fact, a similar large coherent structure can also be found that moves downstream with increasing ambient flow Mach number. This is true for all Mach numbers except for Mach 0.8. For Mach 0.8, the dynamics change completely with running jet as it is shown in fig. 13 . The long coherent structure described above appears to be broken up in several counteracting, more confined coherent structures.

For the applied sign convention, the most dominant coherent structure CSI reflects downstream fluctuations in the reattachment region close to the nozzle exit. This is the same location where the large-scale, clockwise-rotating vortex is typically found, meaning that coherent structure describes in part its dynamic behavior. Further, the same mode captures coherent and interrelated upstream-directed structures along the surface of the nozzle (marked as CS2) and along the initial evolution range of the shear layer (marked as CS3). The latter conveys fluctuations in the shear layer. In other words, the interrelated character between each of the described confined structures suggests a feed-back loop between a large-scale vortex and the excitation of the shear layer. Note that this first POD mode contains about $16 \%$ of the fluctuation energy, while the energy for the second mode still contains $11 \%$ [1].

The decomposition of the velocity field in its modes reveals the most dominant dynamics in the flow field. However, it is sometimes challenging to imagine the impact of a single decomposed mode on the mean velocity field. For this reason, a reduced-order reconstruction of the normalized velocity distribution based on the first POD mode, denoted as $\left(U+\sqrt{2} \sin (\alpha) \zeta_{1, s t d} \Psi_{1}\right) / U_{C}$, is presented for the outstanding Mach 0.8-case with jet for one presumed periodic cycle in fig. 14 The reconstruction was executed with a representative temporal coefficient of $\zeta=\sqrt{2} \sin (\alpha) \zeta_{\text {std }}$. The angle $\alpha$ describes the phase of a POD cycle with $\alpha=90^{\circ}$ and $270^{\circ}$ being the first and second reversal points from the mean 


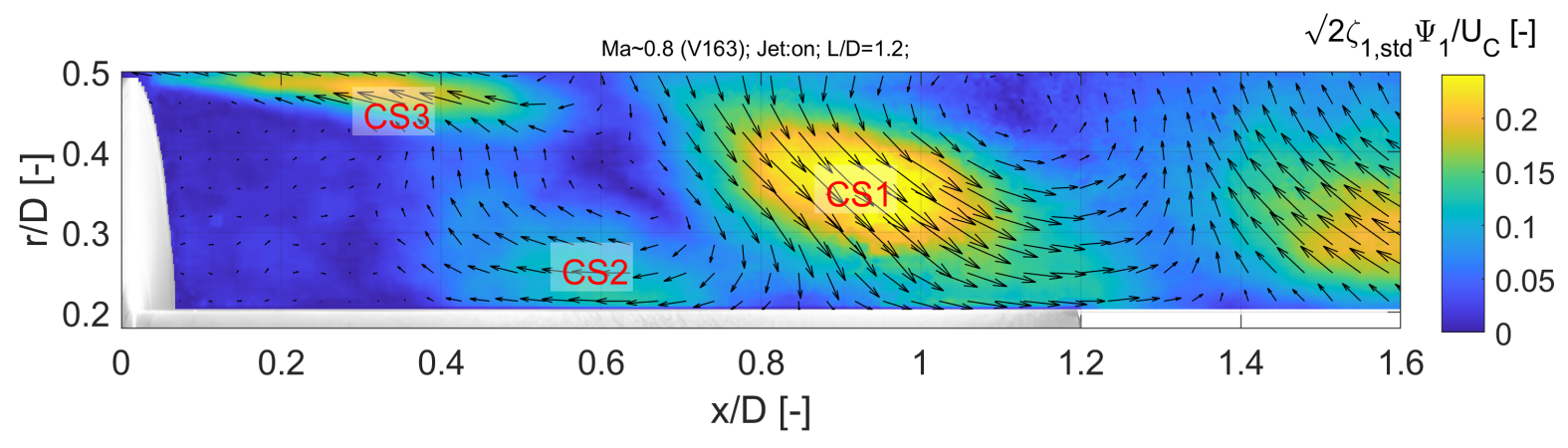

Fig. 13 First POD-decomposed velocity modes (basis function $\Psi_{1}$ scaled with the standard deviation of the time varying coefficient $\zeta_{1, s t d}$ ) for the supersonic exhaust jet configuration with a relative nozzle length $L / D$ of 1.2 at an ambient Mach flow at 0.8 [1]. The vector field and the scalar field show the components of the POD-decomposed velocity fluctuation and their absolute value, respectively.

velocity distribution at $\alpha=0^{\circ}$ and $\alpha=180^{\circ}$. One POD cycle is completed when $\alpha=360^{\circ}=0^{\circ}$ is reached. Further configurations and Mach numbers are discussed in reference [1].

During the first phase $\left(0^{\circ}<\alpha \leq 180^{\circ}\right)$, the recirculation bubble retracts axially and widens in the radial direction. Then in the second phase $\left(180^{\circ} \leq \alpha<360^{\circ}\right)$, the movement is reversed, meaning the bubble is elongated and stretched in the axial direction. As previously indicated by the high-speed schlieren sequence in fig. 10, this appears to result in a vortex shedding mechanism in the phase between $225^{\circ} \leq \alpha<315^{\circ}$. The strongest evidence for that is found for $\alpha=270^{\circ}$. At this point of the cycle, the reconstruction appears to describe the roll-up of a separating vortex. This presumable roll-up and vortex shedding mechanism is also notable to a certain extent for other Mach numbers between 0.5 and 0.9 [1]. Here however, it appears to be substantially amplified. Thus, it raises the question if and how this mechanism plays a role leading to the outstanding turbulence intensity level found above.

\section{Summary and Discussion}

To assess the question how the roll-up and vortex shedding might influence the turbulence intensity, a schematic model concept is proposed. It is a synthesis of the observation attributed to the near-wake dynamics and the jet noise generation mechanism. In detail, it is a synthesis of the findings for the most energetic mode as a result of the POD analysis, the jet screech concept and the finding regarding the symmetry of the incoming pressure waves in combination with the results from literature by Statnikov et al. [5]. First however, a summary of the selected anomalies of the Mach 0.8-case with running jet is given below.

Anomalies can be found in various quantities:

- The base pressure-series in fig. 8 provide the most convincing argument for the presence of special conditions at Mach 0.8 with running jet: There, a distinct sinusoidal-like oscillation can be found which is not observable when the jet is turned off, for higher or for lower Mach numbers. 

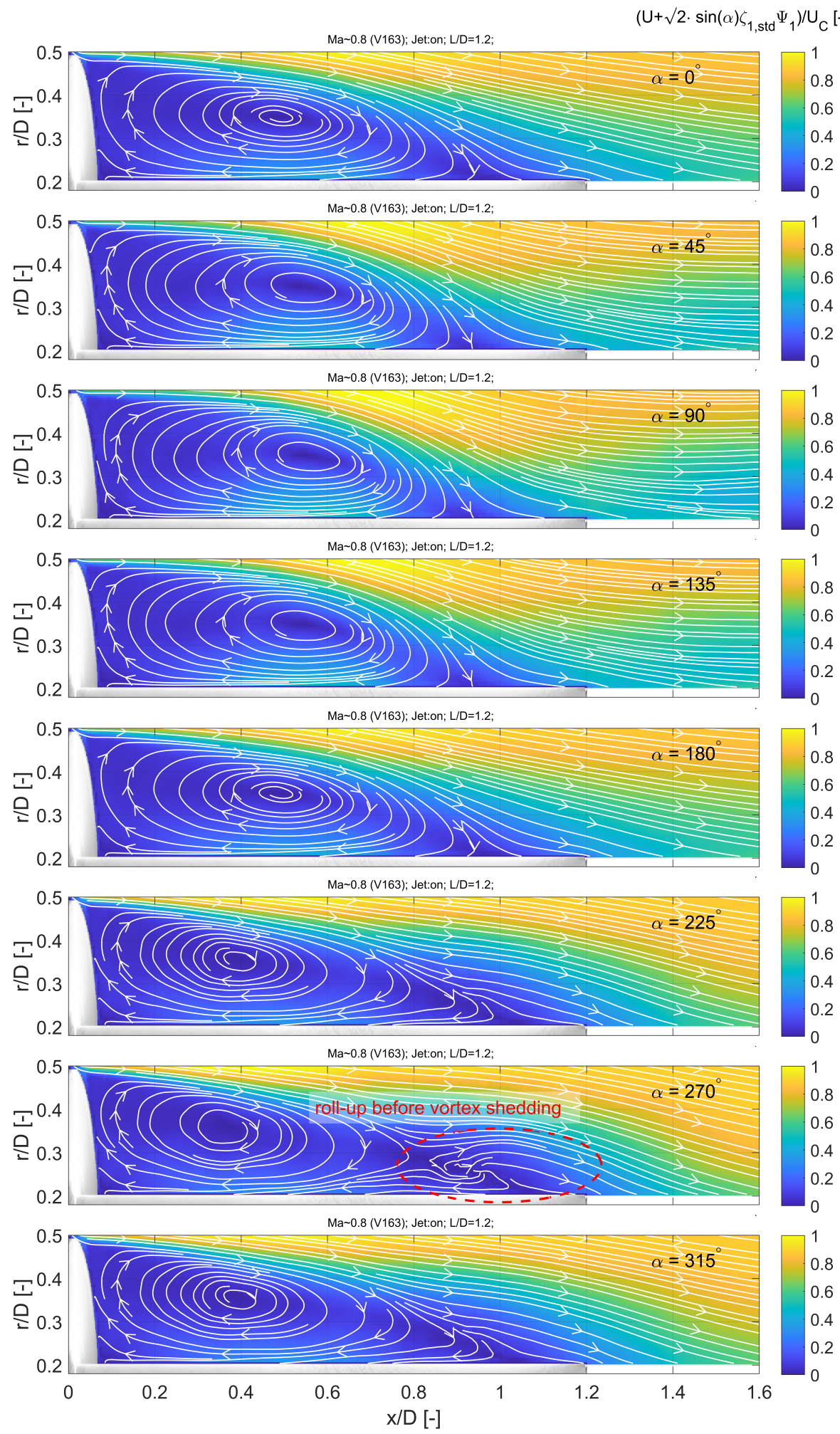

Fig. 14 Scalar field and streamlines of the reconstructed oscillation cycle based on the first POD mode for the supersonic exhaust jet configuration with a relative nozzle length $L / D$ of 1.2 at an ambient Mach number of 0.8 (V163) [1]. 
- Methodologically independent from the base pressure measurements are the velocimetry measurements. They revealed unusually strong (relatively seen) fluctuations in the back flow region of the recirculation bubble (figs. 5 and 6. This correlates with the finding that the shear layer reattachment process becomes hybrid for that Mach number (fig. 77).

- The POD analysis of the PIV data offered insights from a different perspective. A distinguishing redistribution of the fluctuating energy in favor for the first modes is reported in Saile [1] for the Mach 0.8-case with jet. Further, the corresponding first POD seems to be outstanding in comparison between the jet-off and jet-on configuration (fig. 12 vs. fig. 13) and in context to the runs at the other Mach numbers between 0.5 and 0.9 [1]. Next, for the jet-on configuration, the low-order reconstructed cycle (fig. 14p evidences an unusually pronounced roll-up and vortex shedding from the end of the nozzle surface. Last, the probability density function of the temporal coefficients indicated characteristic features for a periodic motion [1]. In summary, the base flow dynamics appears completely altered and amplified when the jet is turned on at Mach 0.8 .

- The roll-up and vortex shedding can also be observed in the sequence of the high-speed schlieren measurements in fig. 10 It appears that this phenomenon is interrelated with an upstream traveling acoustic wave (fig. 9), which is typically associated with the screeching jet noise generation mechanism.

- The spectral analysis performed for both temporally resolving measurements, namely for the pressure and schlieren measurements, revealed matching frequencies peaks (fig. 11). Noteworthy here is the strong pressure amplitude increase when the jet is turned on. This power spectral density peak is about an order of a magnitude larger than for the other Mach-number-cases. Further, the pressure coefficients can be found at a significantly higher level [1]. These anomalies are accompanied with two peculiarities:

- First, the near-wake dynamics without jet features a peak in the spectrum at $S r_{D}=0.35$ (fig. 11). Additionally, in-flight screeching for the same ambient conditions now with a running jet predicts a dominant frequency oscillating with the same frequency, which was then confirmed by measurements. Thus, it was suggested above and it is argued here that the near-wake flow is in resonance with the aeroacoustics of the jet. In detail, there is a positive feed-back loop between the swinging motion of the shear layer and the jet screech both acting at $S r_{D}=0.35-0.36$ for the case at hand.

- Second, for the case without jet [1], the mean reattachment length is located just at the dummy nozzle end. For the case with jet, the shear layer reattachment is still taking place in the vicinity of the nozzle exit and it is shown in fig. 7 that the shear layer is temporary interacting with the jet. The reattachment of the shear layer in the vicinity of the nozzle exit close to the jet appears to promote a pronounced vortex shedding mechanism as evidenced by the schlieren and POD results in fig. 10, and fig.[14, respectively.

Based on these observations, a schematic model concept is suggested in the following. Please note that no claims are raised that the presented schematic is accurate with respect to the temporal sequence between the near-wake dynamics 
and the jet noise generation mechanism. For instance, what is proposed in the following, however cannot be confirmed with the given data set, is the shedding of the vortex in the moment when the pressure wave arrives at the base. Thus, the graph introduced next intends to visualize key elements instead which are believed to play an important role for the anomaly.

The model concept is shown in fig. 15. It depicts a self-exciting cycle, which is divided into four interdependent phases. The sound wave generation phase (1) corresponds to the mechanism as found for screeching. A turbulent structure in the shear-layer generates a pressure wave while passing by the shock-cells. The pressure wave radiates in all directions. Relevant for the cycle is the upstream propagating wave front (2). The pressure wave triggers an excitation in the shear layer such that the recirculation bubble grows until a vortex is shed from the nozzle end (3). The vortex is then fed as turbulent structure in the shear layer of the jet (4). This idea is essential for the coupling of the near-wake and screech dynamics, ergo for the closure of the feed-back loop, since the same turbulent structure finally generates the new pressure wave (1). In other words, this self-sustaining feedback loop is similar to the screech tone excitation mechanism, but instead of an aeroacoustic feed-back at the tip of the nozzle, the vortex shed from the recirculation bubble is fed back as disturbance into the cycle.

The upstream traveling pressure waves are sketched as symmetrically arriving disturbance at the base plate to correspond to the findings regarding the non-existent phase difference for the screech tone [1]. Note that the symmetry of the upstream traveling wave was found for the conditions at hand. Nevertheless, depending on the conditions, the excitation might also take place as an anti-symmetric mode as it can occur for the two suggested driving mechanism, namely screeching [38, 57] and the near-wake dynamics.

The results above indicate that two criteria must be given for a corresponding amplification of the base flow dynamics. The first is the matching resonance frequency, and the second, is the nature of the shear layer reattachment. It appears that the nozzle must be long enough to enable a hybrid shear layer reattachment process. Remember that no such excitation was found for the overshooting shear layer flow with the short $(L / D=0.45)$ nozzle [1].

The question now is if this can also occur in flight for Ariane 5. This question was approached with system analysis-based trajectory data as provided by the DLR Institute of Space Systems. The frequencies for base flow modes swinging, flapping and pumping for the wake flow excitation are simply computed by means of the definition of Strouhal number (eq. (17). Further, the in-flight screeching frequency during the ascent is given by eq. (2) with the convective velocity based on eq. (3) and the velocity of the jet of $4324 \mathrm{~ms}^{-1}$. The latter is calculated for the Ariane 5+Vulcain 2 configuration by means of Ponomarenko [58]. The only parameter left is the shock cell length. This property is not known to the authors. Instead, the shock cell length for the various altitudes is approximated with eq. (4) for nozzle shape parameters given by Seiner and Norum [44], Wolf [9] and as found for the nozzle applied here. Note that all of the corresponding nozzle shape parameters are for cold nozzle flows.

The evolution of the near-wake flow and screeching frequency during the ascent of Ariane 5 is plotted in fig. 16 
(1) SOUND WAVE GENERATION

acoustic

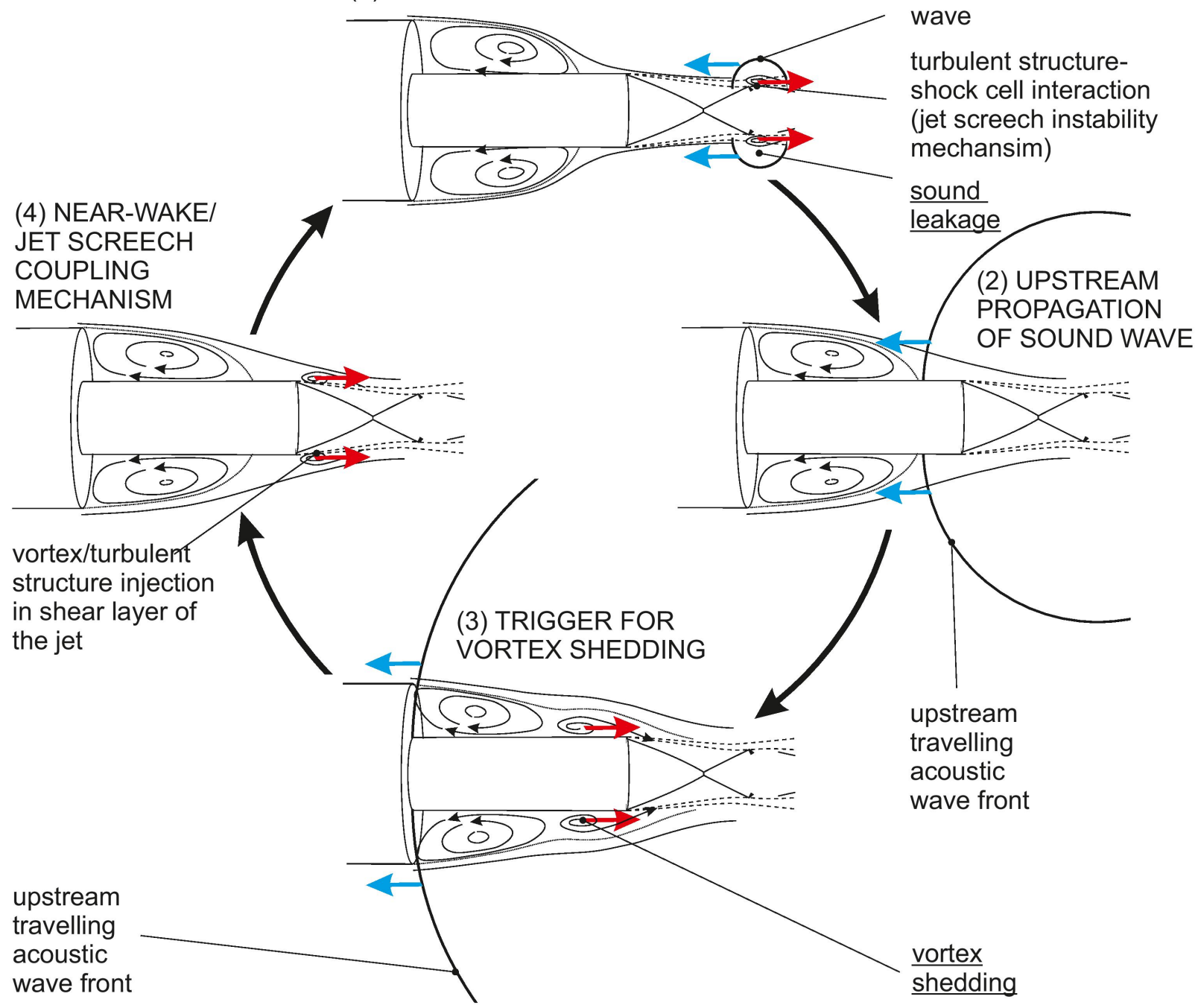

Fig. 15 Synthesis of the current results for the visualization of the hypothetical aeroacoustic coupling between jet screech and the near-wake dynamics [1]. 
as function of the Mach number. The near-wake flow frequencies scale with the ambient flow velocity, and thus increase continuously. On the other hand, the screeching frequency continuously decreases since the upstream traveling disturbance has to move against an accelerating ambient velocity (eq. (2)) until finally at Mach 1 no disturbance from downstream reaches the base anymore. As a result, both mechanisms must exhibit the same frequency at one point in the subsonic flow range, which is true for all near-wake modes. These are the resonance points where an amplifying interaction is hypothesized.

Interestingly, the current simple approach predicts resonance in the ambient Mach number range for which David and Radulovic [3] reports of very strong rms loads on the actuators during the ascent of Ariane 5. The dominant excitation is described to oscillate with a dominant Strouhal number of 0.2. They arise and disappear in the vicinity of Mach 0.8. Further, the actuator is just one element for which strong oscillations were detected. For instance, they are also present in the base pressure measurements [3]. In conclusion, the aeroacoustic interaction hypothesis between the near-wake flow and screeching as suggested in context of fig. 15 provides a plausible explanation for the strong buffeting effects detected for Ariane 5 in the vicinity of Mach 0.8 .

\section{Conclusion}

The current study reviews the hypothesis on an aeroacoustic coupling phenomenon between the near-wake dynamics and the jet screech of space transportation system as reported in Saile [1] or follow-up publications [18--20]. This hypothesis is deduced from anomalies found in wind tunnel tests. In detail, they were found in the data of the various measurement techniques for a space transportation configuration with jet exposed to an ambient Mach-0.8-flow. Anomalies such as the described periodically oscillating base pressure signal, the pronounced turbulence in the base region, or the evidenced shedding mechanism are found by means of a POD decomposition and high-speed schlieren measurements. Further, it is shown that the jet screeching frequency for the configuration with jet occurs at a comparable spectral range as the near-wake flow excitation attributed to swinging for the configuration without jet. The latter observation is taken as an evidence for a resonating case. This resonating case, on the one hand, and the hybrid reattachment process, on the other hand, are assumed as prerequisite for the suggested self-exciting aeroacoustic coupling phenomenon. The corresponding approach was applied on an Ariane 5 ascent, and despite its simplicity, a surprisingly good correlation was found with flight data, in particular with respect the excitations in the base region. Furthermore, it is shown that resonance between the near-wake dynamics and screeching must occur during the ascent of space transportation systems. Thus, the study aims to contribute to the question why dynamic loads are strongly amplified in the high subsonic flow regime.

In the future, it is planned to execute dedicated experiments to further support or falsify the proposed hypothesis. In that context, key features of the two mechanisms in question are in the focus such as the shock cell length, the origin of the aeroacoustic wave and its upstream propagation, the vortex shedding process and the seeding of the turbulent 


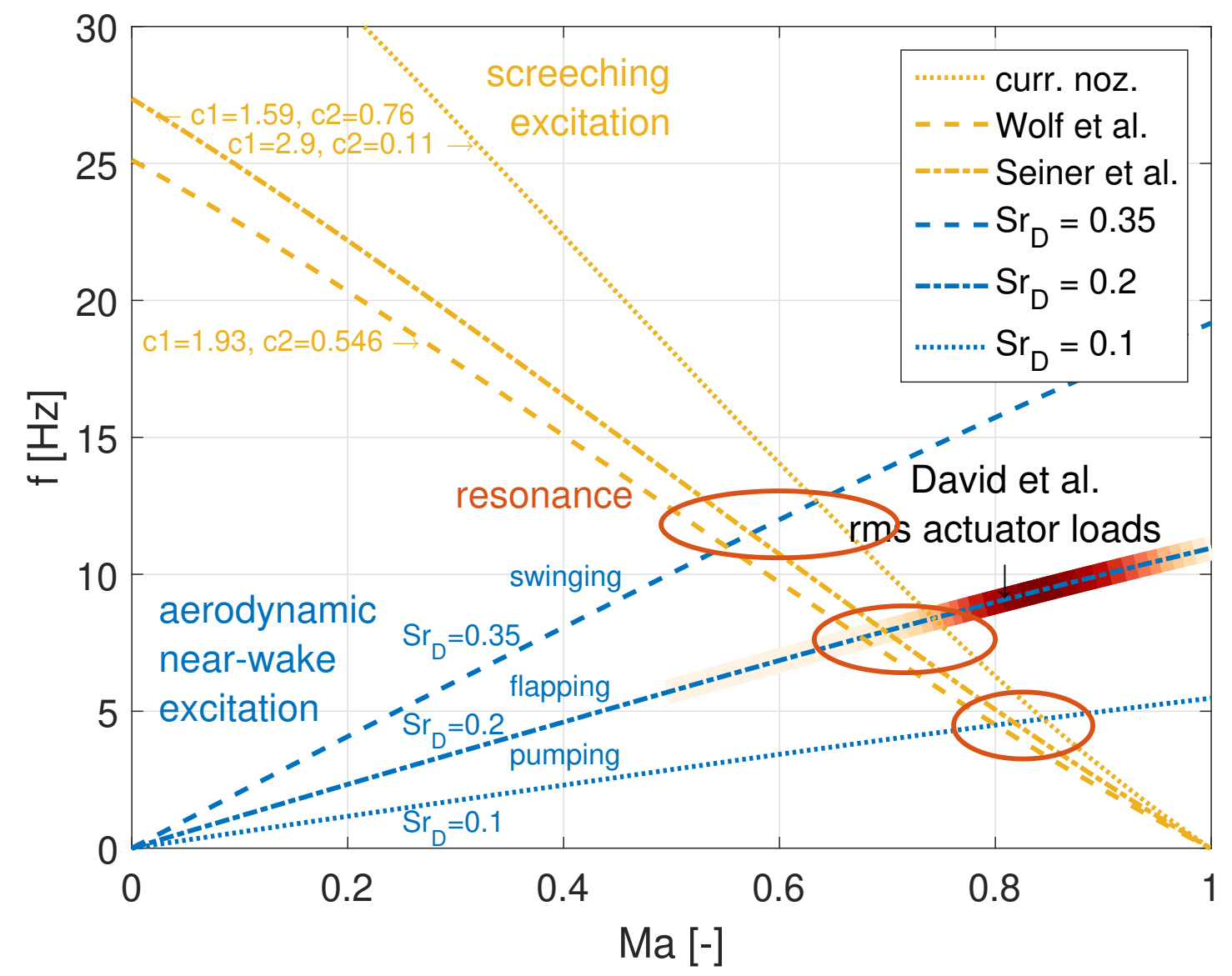

Fig. 16 Evolution of the aerodynamic and screeching excitation frequencies as function of the Mach number in the subsonic flow regime during a representative Ariane 5 ascent and complementary color-coded flight measurements of the Ariane 5 rms actuator loads as given by David and Radulovic [3]. 
structure in the shear layer. In short, the hypothesized cycle and the spatio-temporal dependency between screeching and near-wake dynamics will be scrutinized. Further, it is foreseen to investigate the influence of a flight-realistic, meaning hot jet on that cycle.

\section{Acknowledgments}

Financial support has been provided by the German Research Foundation (Deutsche Forschungsgemeinschaft DFG) in the framework of the Sonderforschungsbereich Transregio 40. Further, thanks a lot to all my colleagues, in particular to the wind tunnel staff, for their support. Last, I want to express my gratitude to Johannes Riehmer and Andreas Flock for their revision and comments and for way more.

\section{References}

[1] Saile, D., "Experimental Analysis on Near-Wake Flows of Space Transportation Systems," Ph.D. thesis, Rheinisch-Westfälische Technische Hochschule Aachen, 2019.

[2] van Gent, P. L., Payanda, Q., Brust, S. G., van Oudheusden, B. W., and Schrijer, F. F. J., "Effects of Exhaust Plume and Nozzle Length on Compressible Base Flows,” AIAA Journal, Vol. 57, No. 3, 2019, pp. 1184-1199. https://doi.org/10.2514/1.J057314. URL https://doi.org/10.2514/1.J057314

[3] David, S., and Radulovic, S., "Prediction of Buffet Loads on the Ariane 5 Afterbody," In 6th Symposium on Launcher Technologies, Munich, Germany, 8-11 November 2005, 2005, pp. 1-12.

[4] Schwane, R., "Numerical Prediction and Experimental Validation of Unsteady Loads on Ariane 5 and Vega," Journal of Spacecraft and Rockets, Vol. 52, No. 1, 2015.

[5] Statnikov, V., Meinke, M., and Schröder, W., "Reduced-Order Analysis of Buffet Flow of Space Launchers," Journal of Fluid Mechanics, Vol. 815, 2017, p. 1-25. https://doi.org/10.1017/jfm.2017.46

[6] Statnikov, V., Bolgar, I., Scharnowski, S., Meinke, M., Kähler, C., and Schröder, W., “Analysis of Characteristic Wake Flow Modes on a Generic Transonic Backward-Facing Step Configuration,” European Journal of Mechanics-B/Fluids, Vol. 59, 2016, pp. 124-134.

[7] Deprés, D., Reijasse, P., and Dussauge, J., “Analysis of Unsteadiness in Afterbody Transonic Flows,” AIAA Journal, Vol. 42, No. 12, 2004, pp. 2541-2550.

[8] Nguyen, T. D., Wells, J. C., Mokhasi, P., and Rempfer, D., "Proper Orthogonal Decomposition-Based Estimations of the Flow Field from Particle Image Velocimetry Wall-Gradient Measurements in the Backward-Facing Step Flow," Measurement Science and Technology, Vol. 21, No. 11, 2010, p. 115406.

[9] Wolf, C. C., "The Subsonic Near-Wake of Bluff Bodies," Ph.D. thesis, Rheinisch-Westfälische Technische Hochschule Aachen, Fakultät für Maschinenwesen, 2013. 
[10] Schrijer, F., Sciacchitano, A., and Scarano, F., "Spatio-Temporal and Modal Analysis of Unsteady Fluctuations in a High-Subsonic Base Flow," Physics of Fluids, Vol. 26, No. 8, 2014, p. 086101.

[11] Hannemann, K., Lüdeke, H., Pallegoix, J.-F., Ollivier, A., Lambaré, H., Maseland, J., Geurts, E., Frey, M., Deck, S., Schrijer, F., et al., "Launch Vehicle Base Buffeting-Recent Experimental and Numerical Investigations," 7th European Symposium on Aerothermodynamics, Vol. 692, 2011, p. 102.

[12] Radespiel, R., Glatzer, C., Hannemann, V., Saile, D., Scharnowski, S., Windte, J., Wolf, C., and You, Y., "SFB Transregio 40: Heckströmungen," Deutscher Luft-und Raumfahrtkongress (DLRK), 2011, pp. 195-205.

[13] Lê, T.-H.-H., "Étude expérimentale du Couplage entre l'Écoulement Transsonique d’Arrière-Corps et les Charges Latérales dans les Tuyères Propulsives," Ph.D. thesis, Université de Poitiers, École Nationale Supérieure de Mécanique et d'Aérotechnique, 2005 .

[14] Schrijer, F., Sciacchitano, A., Scarrano, F., Hannemann, K., Pallegoix, J.-F., Maseand, J., and Schwane, R., "Experimental Investigation of Base Flow Buffeting on the Ariane 5 Launcher using High Speed PIV," 7th European Symposium on Aerothermodynamics, Vol. 692, 2011, p. 103.

[15] Gentile, V., Schrijer, F. F. J., van Oudheusden, B. W., and Scarano, F., "Afterbody Effects on Axisymmetric Base Flows," AIAA Journal, Vol. 54, No. 8, 2016, pp. 2285-2294. https://doi.org/10.2514/1.J054733. URL https://doi.org/10.2514/1.J054733

[16] Gentile, V., Schrijer, F., van Oudheusden, B., and Scarano, F., "Very-Low Frequency Wake Dynamics of an Axisymmetric Body," International Workshop on Non-Intrusive Optical Flow Diagnostics, 2016, pp. 1-5.

[17] van Gent, P., Schrijer, F., and van Oudheusden, B., "Assessment of the Pseudo-Tracking Approach for the Calculation of Material Acceleration and Pressure Fields from Time-Resolved PIV: Part II. Spatio-Temporal Filtering," Measurement Science and Technology, Vol. 29, No. 4, 2018, p. 045206.

[18] Saile, D., Kühl, V., and Gülhan, A., “On the Subsonic Near-Wake of a Space Launcher Configuration without Jet,” Experiments in Fluids, Vol. 60, No. 4, 2019, p. 50. https://doi.org/10.1007/s00348-019-2690-9. URL/https://doi.org/10.1007/s00348-019$2690-9$

[19] Saile, D., Kühl, V., and Gülhan, A., "On the Subsonic Near-Wake of a Space Launcher Configuration with Exhaust Jet," Experiments in Fluids, Vol. 60, No. 11, 2019, p. 165. https://doi.org/10.1007/s00348-019-2801-7. URL https: //doi.org/10.1007/s00348-019-2801-7

[20] Saile, D., Kühl, V., and Gülhan, A., "On Subsonic Near-Wake Flows of Various Base Geometries,” 13th International Symposium on Particle Image Velocimetry (ISPIV 2019), July 22.-24., 2019, 2019, pp. 1-14.

[21] Kirchheck, D., Saile, D., and Gülhan, A., "Spectral Analysis of Rocket Wake Flow-Jet Interaction by Means of High-speed Schlieren Imaging," 8th European Conference for Aeronautics and Space Sciences (EUCASS), 2019, pp. 1-4. 
[22] Deck, S., and Thorigny, P., "Unsteadiness of an Axisymmetric Separating-Reattaching Flow: Numerical Investigation,” Physics of Fluids, Vol. 19, No. 6, 2007, p. 065103.

[23] Weiss, P.-É., Deck, S., Robinet, J.-C., and Sagaut, P., "On the Dynamics of Axisymmetric Turbulent Separating/Reattaching Flows," Physics of Fluids, Vol. 21, No. 7, 2009, p. 075103.

[24] Weiss, P.-É., and Deck, S., "Numerical Investigation of the Robustness of an Axisymmetric Separating/Reattaching Flow to an External Perturbation using ZDES," Flow, Turbulence and Combustion, Vol. 91, No. 3, 2013, pp. 697-715.

[25] Pain, R., Weiss, P.-E., and Deck, S., "Zonal Detached Eddy Simulation of the Flow Around a Simplified Launcher Afterbody," AIAA Journal, Vol. 52, No. 9, 2014, pp. 1967-1979. https://doi.org/10.2514/1.J052743, URL https://doi.org/10.2514/1.J052743

[26] Pain, R., Weiss, P.-E., Deck, S., and Robinet, J.-C., "Large Scale Dynamics of a High Reynolds Number Axisymmetric Separating/Reattaching Flow," Physics of Fluids, Vol. 31, No. 12, 2019, p. 125119.

[27] Banuti, D., “TRP on Hot Plume Testing Facilities for ELV Propulsion Characterization, WP2200: Scaling Issues,” Ref.: Proposal-No. 3005921, 2013.

[28] Morkovin, M., "Flow Around Circular Cylinder - A Kaleidoscope of Challenging Fluid Phenomena," In Haswen, A.G. (Ed.): Symposium of Fully Separated Flows, 1964, pp. 102-118.

[29] Roshko, A., and Lau, J. C., "Some Observations on Transition and Reattachment of a Free Shear Layer in Incompressible Flow," Proc. 1965 Heat Transfer and Fluid Mechanics Institute, edited by AF Charwat, Stanford University Press, Stanford, 1965, pp. $157-167$.

[30] Westphal, R. V., Johnston, J., and Eaton, J., "Experimental Study of Flow Reattachment in a Single-Sided Sudden Expansion," NASA Contractor Report 3765, 1984.

[31] Merz, R., Page, R., and Przirembel, C., “Subsonic Axisymmetric Near-Wake Studies,” AIAA Journal, Vol. 16, No. 7, 1978.

[32] Adams, E. W., and Johnston, J. P., "Effects of the Separating Shear Layer on the Reattachment Flow Structure; Part 1: Pressure and Turbulence Quantities," Experiments in Fluids, Vol. 6, No. 6, 1988, pp. 400-408.

[33] Nadge, P. M., and Govardhan, R., "High Reynolds Number Flow over a Backward-Facing Step: Structure of the Mean Separation Bubble," Experiments in Fluids, Vol. 55, No. 1, 2014, p. 1657.

[34] Goethert, B., and Barnes, L., "Some Studies of the Flow Pattern at the Base of Missiles with Rocket Exhaust Jets," Tech. rep., DTIC Document, 1960.

[35] Tam, C. K., “Supersonic Jet Noise,” Annual Review of Fluid Mechanics, Vol. 27, No. 1, 1995, pp. $17-43$.

[36] Raman, G., “Advances in Understanding Supersonic Jet Screech: Review and Perspective,” Progress in Aerospace Sciences, Vol. 34, No. 1, 1998, pp. 45-106. 
[37] Raman, G., "Supersonic Jet Screech: Half-Century from Powell to the Present," Journal of Sound and Vibration, Vol. 225, No. 3, 1999, pp. 543-571.

[38] Powell, A., "On the Mechanism of Choked Jet Noise," Proceedings of the Physical Society. Section B, Vol. 66, No. 12, 1953, p. 1039.

[39] Powell, A., "On the Noise Emanating from a Two-Dimensional Jet above the Critical Pressure," Aeronautical Quarterly, Vol. 4, No. 02, 1953, pp. 103-122.

[40] Tam, C., Seiner, J., and Yu, J., "Proposed Relationship between Broadband Shock Associated Noise and Screech Tones," Journal of Sound and Vibration, Vol. 110, No. 2, 1986, pp. 309-321.

[41] Manning, T., and Lele, S., “A Numerical Investigation of Sound Generation in Supersonic Jet Screech,” 6th Aeroacoustics Conference and Exhibit, 2000, p. 2081.

[42] Suzuki, T., and Lele, S., "Shock Leakage through an Unsteady Vortex-Laden Mixing Layer-Application to Jet Screech,” 7th AIAA/CEAS Aeroacoustics Conference and Exhibit, 2001, p. 2141.

[43] André, B., Castelain, T., and Bailly, C., "Experimental Study of Flight Effects on Screech in Underexpanded Jets," Physics of Fluids, Vol. 23, No. 12, 2011, p. 126102.

[44] Seiner, J., and Norum, T., “Aerodynamic Aspects of Shock Containing Jet Plumes,” 6th Aeroacoustics Conference, 1980 , p. 965.

[45] Hay, J., and Rose, E., “In-Flight Shock Cell Noise,” Journal of Sound Vibration, Vol. 11, 1970, p. 411.

[46] Bryce, W., and Pinker, R., "The Noise from Unheated Supersonic Jets in Simulated Flight,” 4th Aeroacoustics Conference, 1977, p. 1327.

[47] Adrian, R., "Dynamic Ranges of Velocity and Spatial Resolution of Particle Image Velocimetry," Measurement Science and Technology, Vol. 8, No. 12, 1997, p. 1393.

[48] Jordan, P., and Colonius, T., "Wave Packets and Turbulent Jet Noise," Annual Review of Fluid Mechanics, Vol. 45, 2013 , pp. 173-195.

[49] Poldervaart, L., Vink, A., and Wijnands, A., "Jet Screech,” Film produced for Aerodynamic Division of the Physics Department, G. Vossers, Technological University of Eindhoven, 1969. URL https://www.youtube.com/watch?v=dJp5m1SDN5c

[50] Poldervaart, L., Wijnands, A., van Moll, L., and van Voorthuisen, E., "Modes of Vibration of Two-Dimensional Jet of Air," Film produced by University of Technology of Eindhoven, Physic Department - Aerodynamic Group, Netherlands, 1974. URL https://www.youtube.com/watch?v=EnlCDOQW7wc.

[51] Triesch, K., and Krohn, E.-O., Die Vertikale Meßstrecke der DFVLR in Köln-Porz (Stand 1986), DFVLR-Mitt. 86-22, Wissenschaftliches Berichtswesen der DFVLR, ISSN 0176-7739, Postfach 906058, 5000 Köln 90, 1986. 
[52] Saile, D., Kirchheck, D., Gülhan, A., and Banuti, D., "Design of a Hot Plume Interaction Facility at DLR Cologne," Proceedings of the 8th European Symposium on Aerothermodynamics for Space Vehicles, 2015, pp. 1-17.

[53] Tropea, C., and Yarin, A. L., Springer Handbook of Experimental Fluid Mechanics, Vol. 1, Springer Science \& Business Media, 2007.

[54] Welch, P., "The Use of Fast Fourier Transform for the Estimation of Power Spectra: A Method Based on Time Averaging over Short, Modified Periodograms," IEEE Transactions on Audio and Electroacoustics, Vol. 15, No. 2, 1967, pp. 70-73.

[55] Sirovich, L., "Turbulence and the Dynamics of Coherent Structures. I. Coherent Structures," Quarterly of Applied Mathematics, Vol. 45, No. 3, 1987, pp. 561-571.

[56] Uberoi, M. S., and Freymuth, P., "Turbulent Energy Balance and Spectra of the Axisymmetric Wake," The Physics of Fluids, Vol. 13, No. 9, 1970, pp. 2205-2210.

[57] Norum, T., "Screech Suppression in Supersonic Jets," AIAA Journal, Vol. 21, No. 2, 1983, pp. 235-240.

[58] Ponomarenko, A., "Rocket Propulsion Analysis," , 2019. URL http://propulsion-analysis.com/index.htm 